This manuscript is a non-peer reviewed EarthArXiv preprint that has been submitted for publication in Continental Shelf Research. If accepted, the final version of this manuscript will be available via the 'Peerreviewed Publication DOI' link on the right-hand side of this webpage. 


\title{
Non-deterministic effects in modelling the tidal currents in a high-energy coastal site
}

\author{
Simon C. Warder ${ }^{\mathrm{a}, *}$, Stephan C. Kramer ${ }^{\mathrm{a}}$, Matthew D. Piggott ${ }^{\mathrm{a}}$ \\ ${ }^{a}$ Department of Earth Science and Engineering, Imperial College London, London, SW7 2AZ, UK
}

\begin{abstract}
Numerical models are commonly employed for predicting tidal stream resource, since harmonic analysis is typically insufficient for representing tidal currents. This is particularly true in regions of high-energy flow and complex bathymetric and coastline features, resulting in highly nonlinear dynamics. Within this work, we demonstrate that nondeterministic effects pose a further barrier to resource predictability in such regions. We take the Inner Sound of the Pentland Firth as a case study region, situated between mainland Scotland and the island of Stroma, where nondeterministic effects are introduced by eddy shedding off islands and headlands in the region. We set up a numerical model of the region using the Thetis finite element coastal ocean model. We first demonstrate the presence of nondeterministic effects, by forcing the model with a single tidal constituent and observing the departure from purely periodic behaviour within the Inner Sound, and also by comparing an ensemble of fully-forced model runs generated with perturbed initial conditions. We then take steps towards model calibration with respect to the model bottom friction and viscosity parameters, comparing a variety of choices of model-observation error metrics for this purpose. The non-deterministic component not only places a limit on the predictability of the flow (and hence the tidal stream resource), but also on the calibration process. Focusing on calibration with respect to the bottom friction parameter, we quantify the identifiability limit which the non-deterministic behaviour places on the parameter. We explore the sensitivity of this identifiability limit with respect to the viscosity parameter and the choice of error metric. We find that calibration based on matching the modelled and observed mean kinetic power density is a good choice, and is relatively robust with respect to the non-deterministic behaviour. Based on these results, and on the sensitivity of the error metrics to each model parameter and the mesh resolution, we make recommendations for how to proceed with model calibration in the presence of non-deterministic flow. Since the non-deterministic effect is due to complex, high-energy flow leading to highly nonlinear dynamics, we expect these results to generalise to other regions of high tidal stream resource.
\end{abstract}

Keywords: Predictability; Non-deterministic behaviour; Model calibration; Tidal stream; Pentland Firth

${ }^{*}$ Corresponding author

Email addresses: s.warder15@imperial.ac.uk (Simon C. Warder), s.kramer@imperial.ac.uk (Stephan C. Kramer), m.d.piggott@imperial.ac.uk (Matthew D. Piggott) 


\section{Introduction}

The seas to the north of the Scottish mainland have high potential for renewable energy generation, with a number of areas leased for tidal stream energy extraction (Neill et al., 2017, Marine Scotland, 2015, Crown Estate, 2013). The Pentland Firth, situated between mainland Scotland and the Orkney Islands, connects the North Atlantic at its western end to the North Sea at its eastern end, resulting in a large tidal phase difference along its length (Easton et al., 2012). This high phase difference is responsible for the high-energy flow within the Pentland Firth (O'Hara Murray and Gallego, 2017); its tidal stream resource has been estimated by various sources to be between 0.62 and $9 \mathrm{GW}$ (ABPmer, 2007, MacKay, 2008, Adcock et al., 2013, Kreitmair et al., 2019), with most of this variation attributed to differing modelling approaches, assumptions and methodologies (Kreitmair et al., 2020). The Inner Sound of the Pentland Firth, separated from the main strait by the island of Stroma, contains a site leased to MeyGen for the extraction of up to 400 MW (Crown Estate, 2012). Draper et al. (2014) subsequently estimated the tidal stream resource of the Inner Sound to be between 108 and $320 \mathrm{MW}$, depending on the operation of other tidal stream extraction sites across the wider Pentland Firth. Due to its renewable energy resource and highly energetic dynamics, the Pentland Firth, and in particular the Inner Sound, are of great practical and theoretical interest, resulting in significant attention from both a modelling (Easton et al., 2012, Gillibrand et al., 2016, O'Hara Murray and Gallego, 2017, Rahman and Venugopal, 2017, Avdis et al., 2018) and data acquisition (Shields et al., 2009, Goddijn-Murphy et al., 2013) perspective.

A significant aspect of the appeal of tidal renewable energy is its predictability. The use of harmonic analysis for the prediction of tidal elevations is well established, and in many cases currents can be similarly treated by assuming the presence of periodic components dictated by astronomical forcing. However, in practice, coastal ocean currents are subject to variations on relatively small scales, due primarily to coastal geometry and bathymetry, which cannot be captured by harmonic analysis. Such non-harmonic flow can arise from several sources, such as density-driven circulation, flow separation, eddies, or other non-linear features (Polagye et al., 2010, Adcock et al., 2015), which may be particularly prevalent in flows with high velocities. In the Pentland Firth, local geographical features and highenergy flow result in extremely complex tidal dynamics (Easton et al., 2012, Goddijn-Murphy et al., 2013). As a result, tidal stream resource predictability typically relies on numerical modelling, although due to modelling assumptions, finite model resolution and imperfect model inputs, there remain practical limitations to accurately predicting the energy resource (Adcock et al., 2015). As we will show within this paper, these limitations are compounded by non-deterministic effects arising due to eddy shedding within the high-energy flow.

An aspect of numerical models which requires particular attention is the treatment of subgrid-scale processes. Within both 3D and depth-averaged coastal ocean models, this typically includes parameterisations for friction between the ocean and sea floor, and for unresolved turbulence. Common formulations for bottom friction include linear or quadratic relationships between the frictional force and the ocean current (Zhang et al., 2011), while unresolved turbulence can be modelled by a variety of closure schemes (Baston et al., 2013). In each case, at least one tunable parameter is introduced to the model, which must be appropriately selected, typically via a model calibration procedure. As well as exhibiting increased spatial variation compared with the surface elevation, modelled velocities are typically also more sensitive to such tuning parameters, further increasing uncertainty in tidal resource assessment via numerical models, and placing a requirement on high-quality observation data and robust calibration procedures. The bottom friction parameter has been found to be particularly influential in accurately capturing the dynamics within the 
Pentland Firth (Rahman and Venugopal, 2015), with $60 \%$ of the energy entering the Pentland Firth estimated to be lost through bottom friction (Easton et al., 2012).

In the context of the Pentland Firth, there exists a variety of sources of data which can be used for model calibration (O'Hara Murray and Gallego, 2017, Side et al., 2017), and there have been a variety of modelling studies featuring some form of calibration for bottom friction coefficients (e.g. Easton et al. (2012), Wolf et al. (2016), Waldman et al. (2017)). However, the model calibration in each of these studies takes only a simplistic approach, dealing only with uniform friction parameters, and performing calibration by running the model with a range of parameter values and selecting the value which gives the best model performance. This is typically performed using only a small handful of parameter values (often just three), and with only one degree of freedom (i.e. one parameter at a time). In the broader coastal ocean modelling literature there exist a variety of more sophisticated calibration strategies (e.g. gradient-based methods (Heemink et al., 2002, Lu and Zhang, 2006, Maßmann, 2010, Sraj et al., 2014a, Chen et al., 2014), Bayesian inference approaches (Sraj et al., 2013, 2014b) and evolutionary algorithms (Huybrechts et al., 2021)), which to date have not been employed within models of the Pentland Firth. However, the complexity of the dynamics within the Pentland Firth poses an additional challenge to the implementation of such methods; the above calibration strategies have not previously been applied in such a high-energy and complex domain.

As a step towards a more sophisticated model calibration, in this study we set up a numerical model of the Pentland Firth within the finite element coastal ocean model Thetis, with the aim of quantifying the predictability of the tidal stream resource within the Inner Sound. We find that eddies shed off islands and headlands in the region induce a departure from purely harmonic behaviour, and furthermore that there is a non-deterministic component to the flow. This non-deterministic behaviour introduces an additional barrier to resource predictability and model calibration. The aim of this paper is therefore to demonstrate the existence of this non-deterministic component, and investigate its implications for resource predictability and model calibration.

The numerical model and data sources used within this work are described in section 2 . In section 3 we present model results demonstrating the presence of this non-deterministic component in the Inner Sound. However, the non-deterministic effect is strongly dependent on the value for the viscosity parameter within the numerical model, and can be suppressed through the use of elevated viscosity values (e.g. $\nu=40 \mathrm{~m}^{2} \mathrm{~s}^{-1}$ ). In section 4 we investigate the response of a variety of error metrics (measuring error against observations from an ADCP deployed within the Inner Sound) to the friction and viscosity parameters at a coarse parameter resolution, which enables a simple model calibration. In section 5, we further investigate the limitations imposed on the calibration problem by the nondeterministic component of the flow, and we show that suppressing the non-deterministic behaviour via the use of a high viscosity parameter is beneficial in achieving finer resolution in the friction coefficient via calibration methods. In section 6 we briefly investigate the error metrics as a function of mesh resolution. Here we observe that, while finer resolution achieves overall improved performance, the optimal model parameters are not strongly sensitive to the mesh resolution, and therefore that a practical approach to model calibration is a multi-scale approach, where the calibration is first performed on a coarse mesh, before further parameter fine-tuning on a higher-resolution model as desired. The implications of these findings for tidal resource predictability and model calibration are discussed in section 7 , and we draw conclusions in section 8 . 


\section{Model and data}

\subsection{Numerical model setup}

The numerical model results presented herein are obtained using Thetis, a 3D (Kärnä et al., 2018, Pan et al., 2020) and 2D (Vouriot et al., 2019) finite element coastal ocean model, built using the Firedrake framework (Rathgeber et al., 2016). Used here in its two-dimensional configuration, the model solves the non-linear shallow water equations in the form

$$
\begin{aligned}
\frac{\partial \eta}{\partial t}+\nabla \cdot(H \mathbf{u}) & =0 \\
\frac{\partial \mathbf{u}}{\partial t}+\mathbf{u} \cdot \nabla \mathbf{u}+\mathbf{F}_{\mathbf{C}}+g \nabla \eta & =-\frac{\boldsymbol{\tau}_{b}}{\rho H}+\nabla \cdot\left(\nu\left(\nabla \mathbf{u}+\nabla \mathbf{u}^{T}\right)\right),
\end{aligned}
$$

where $\eta$ is the surface elevation, $H=\eta+h$ the total water depth, $h$ the bathymetry, $\mathbf{u}$ the depth-averaged velocity, $\mathbf{F}_{\mathbf{C}}$ the Coriolis force, $g$ the acceleration due to gravity, $\boldsymbol{\tau}_{b}$ the bottom stress due to friction, $\rho$ the density of seawater, and $\nu$ the eddy viscosity. The bottom stress is parameterised here using Manning's $n$ formulation, given by

$$
\frac{\boldsymbol{\tau}_{b}}{\rho}=\frac{g n^{2}}{H^{\frac{1}{3}}}|\mathbf{u}| \mathbf{u}
$$

where $n$ is the (spatially varying) Manning coefficient. We introduce wetting and drying via the bathymetry modification scheme of Kärnä et al. (2011). The spatial extent of intertidal regions within the Pentland Firth is very small. However, we found through preliminary experiments (not shown) that without employing a wetting-drying scheme, a large minimum water depth was required to achieve model stability across a wide range of Manning coefficients and viscosities. The wetting-drying scheme removes the need for a minimum depth and consequently improves model accuracy. The wetting-drying scheme introduces a new parameter, $\alpha$, which governs the transition from wet to dry regions of the domain. The value for $\alpha$ used throughout this work is $0.01 \mathrm{~m}$. The model outputs show very little sensitivity to $\alpha$, and while the parameter is in principle mesh resolution-dependent, this value was found to perform robustly on all meshes tested.

The meshes used within this work were generated on a UTM30 coordinate projection, using the Python package qmesh (version 1.0.2) (Avdis et al., 2018), which interfaces the mesh generator Gmsh (version 3.0.4) (Geuzaine and Remacle, 2009). The coastline data is from the Global Self-consistent, Hierarchical, High-resolution Geography Database (GSHHG) (Wessel and Smith, 1996). The primary mesh used within this study is shown in figure 1a, which has a resolution of $200 \mathrm{~m}$ throughout the Pentland Firth, $1 \mathrm{~km}$ along the coastlines, and $15 \mathrm{~km}$ in open regions, resulting in a total of 84,772 elements. Section 6 explores the influence of mesh resolution on model outputs, using two additional meshes with further refinement within the Inner Sound of the Pentland Firth (to $100 \mathrm{~m}$ and $50 \mathrm{~m}$, respectively). These refined meshes are also shown in figure 1.

The governing equations (1) are solved using a $\mathrm{P}_{1}^{\mathrm{DG}}-\mathrm{P}_{1}^{\mathrm{DG}}$ finite element pair, and a Crank-Nicolson timestepping scheme with a time step $\Delta t=100 \mathrm{~s}$. Through preliminary numerical experiments (not shown), we found that the model results are insensitive to this choice of time step, and that it is compatible with all meshes used within this study. A Dirichlet boundary condition is applied for the elevation $\eta$ on the ocean boundary, generated from tidal harmonics from the TPXO dataset (Egbert and Erofeeva, 2002). Most model runs within this work use a boundary condition based on all 11 constituents from the database (Q1, O1, P1, K1, N2, M2, S2, K2, M4, MS4, MN4), with some model runs (where stated) forced using the M2 constituent alone. 

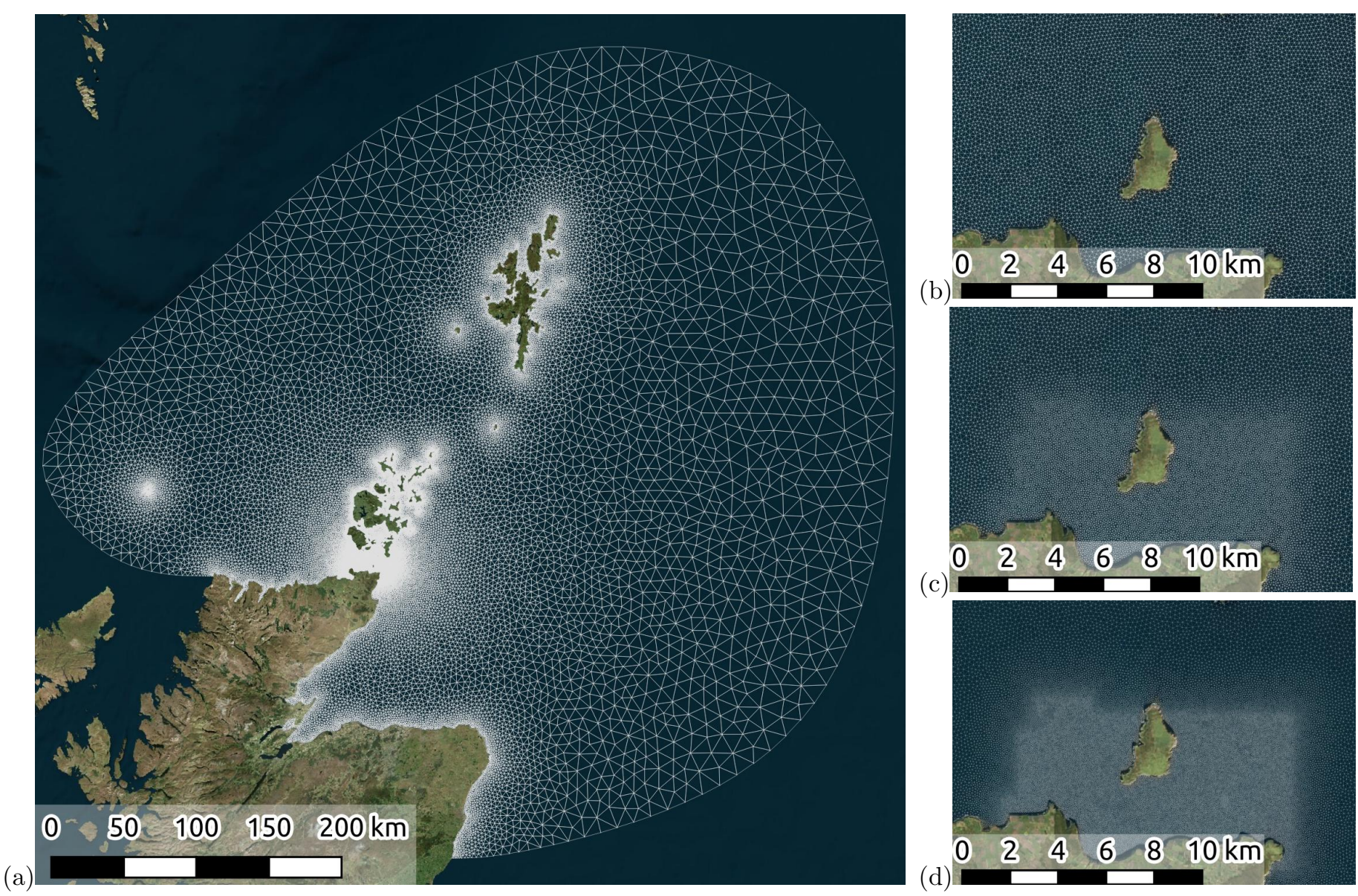

(b)

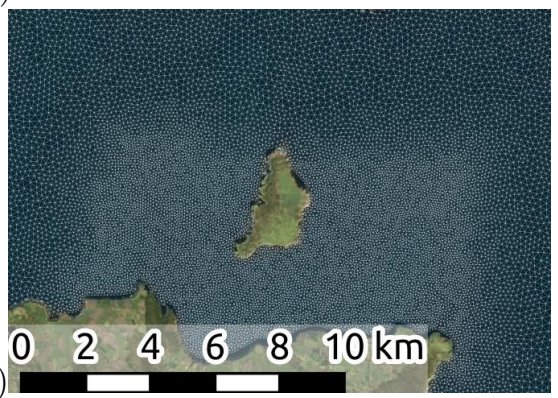

(c)

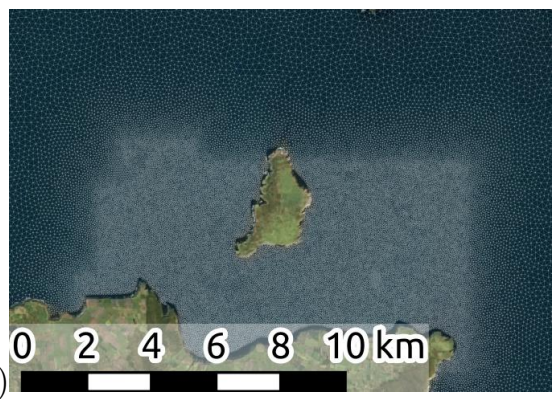

Figure 1: (a) Primary mesh used within this paper. (b) Primary mesh zoomed to Inner Sound, resolution $200 \mathrm{~m}$. (c) $100 \mathrm{~m}$ resolution within Inner Sound. (d) $50 \mathrm{~m}$ resolution within Inner Sound.

\subsection{Bathymetry data sources}

The bathymetry used within this work combines data from several sources, which are indicated in figure 2 and summarised here:

- MeyGen survey. This data covers a small region within the Inner Sound, at a grid resolution of $1 \mathrm{~cm}$, and a datum of -50 m relative to Mean Sea Level (MSL).

- Marine Scotland survey (Marine Scotland, 2013). This data covers most of the Pentland Firth, at a resolution of $2 \mathrm{~m}$, using Lowest Astronomical Tide (LAT) datum.

- Digimap (Digimap, 2013). This data covers the entire model domain. However, due to unrealistic features observed in parts of the dataset (gridding artefacts, and visible joins between source datasets), Digimap data is only used within the polygon indicated in figure 2, within which no such artefacts are visible. The dataset has a resolution of 1 arcsecond $(\sim 30 \mathrm{~m})$, and is provided in LAT datum.

- EMODnet (EMODnet Bathymetry Consortium, 2018). This data covers the entire model domain, at a resolution of $\sim 115 \mathrm{~m}$, using LAT datum.

Each of these sources were first converted to Mean Sea Level (MSL) datum, with the datasets given in LAT datum corrected based on predicted astronomical tidal ranges from the TPXO tidal database used to force the model. The 
MeyGen, Marine Scotland and Digimap datasets were then blended into a single dataset on a uniform $20 \mathrm{~m}$ grid covering the Pentland Firth and the Orkneys. This grid resolution is finer than any mesh used within this study, and this regridding is therefore assumed not to introduce errors. EMODnet data is used outside of this region, with the blending of the high-res and EMODnet datasets handled at the point of interpolation onto the mesh in order to avoid the need for a single high-resolution bathymetry data file covering the whole model domain.
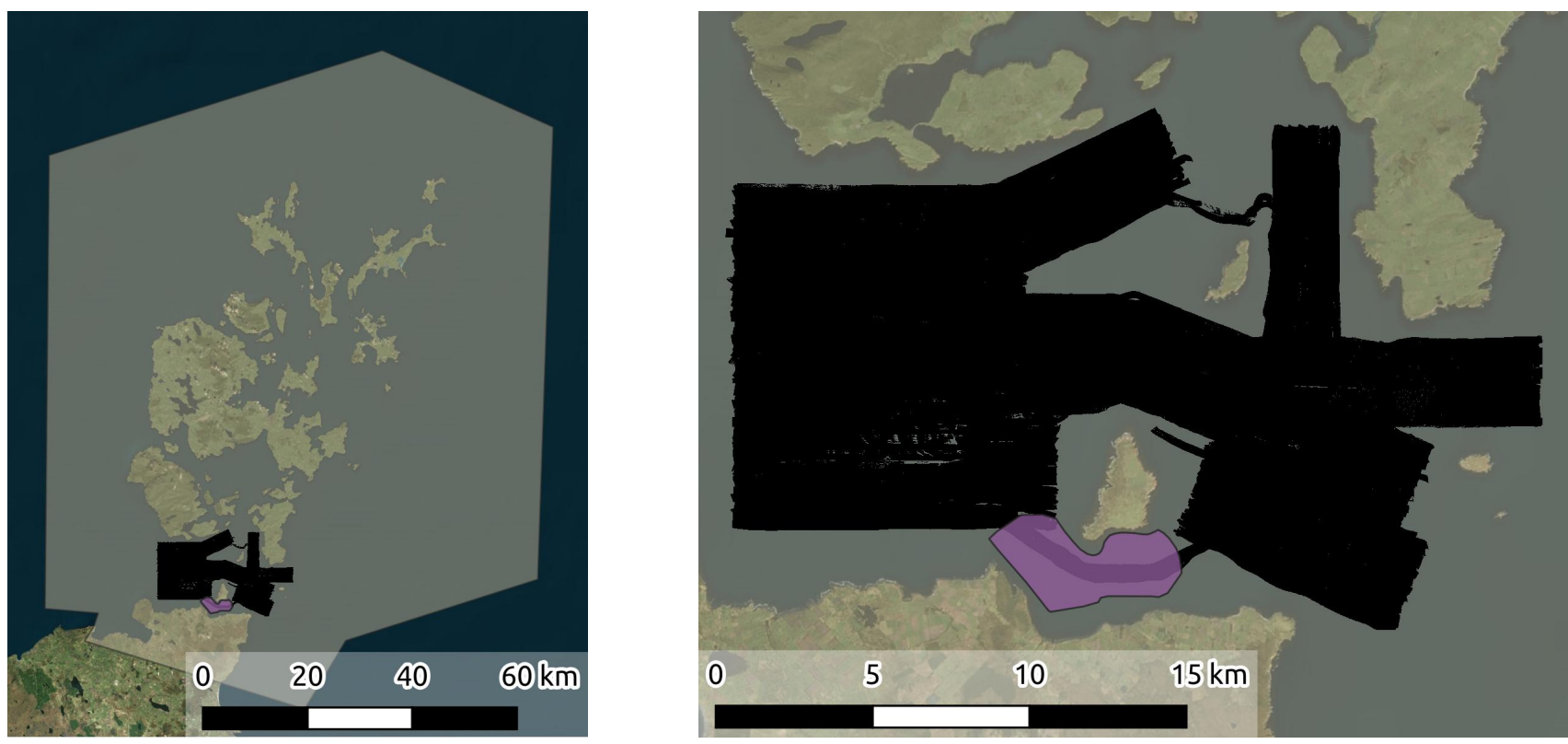

Figure 2: Bathymetry data sources. Purple: MeyGen survey region. Black: Marine Scotland survey region. Outer polygon: region where Digimap bathymetry is trusted. Outside this polygon, EMODnet data is used.

\section{3. $A D C P$ data}

Within this work, we compare model results with velocity observations from an ADCP deployed in the Inner Sound, at the location indicated in figure 3. The ADCP was deployed for 33 days, and we make comparisons with data which have been time-averaged to $10 \mathrm{~min}$ and depth-averaged. We apply the same time-averaging to the model outputs.

Since we are motivated by tidal stream energy, within this work we make comparisons with the ADCP data only when the current speed is in excess of a cut-in velocity of $0.7 \mathrm{~m} \mathrm{~s}^{-1}$. For model-observation comparisons, the $x$ and $y$-components of the velocity are combined into a single velocity scalar, whose sign is given by the sign of the $x$-component of the velocity, i.e.

$$
\text { vel }=\sqrt{u^{2}+v^{2}} \frac{u}{|u|},
$$

where $u$ and $v$ are the $x$ - and $y$-components of the velocity, respectively. The use of only a single (scalar) velocity simplifies model-observation comparisons, and for tidal stream energy the direction is less important than the magnitude, if a turbine is designed to passively yaw (Polagye et al., 2010). The flow through the Inner Sound is primarily east-west (i.e. $|u|>|v|$ ) (Goddijn-Murphy et al., 2013), hence we take the sign of $u$ as the sign of the overall flow (i.e. as an indicator of flood/ebb tide). Departures from east-west dominated flow only occur below the cut-in velocity, i.e. at slack water. 


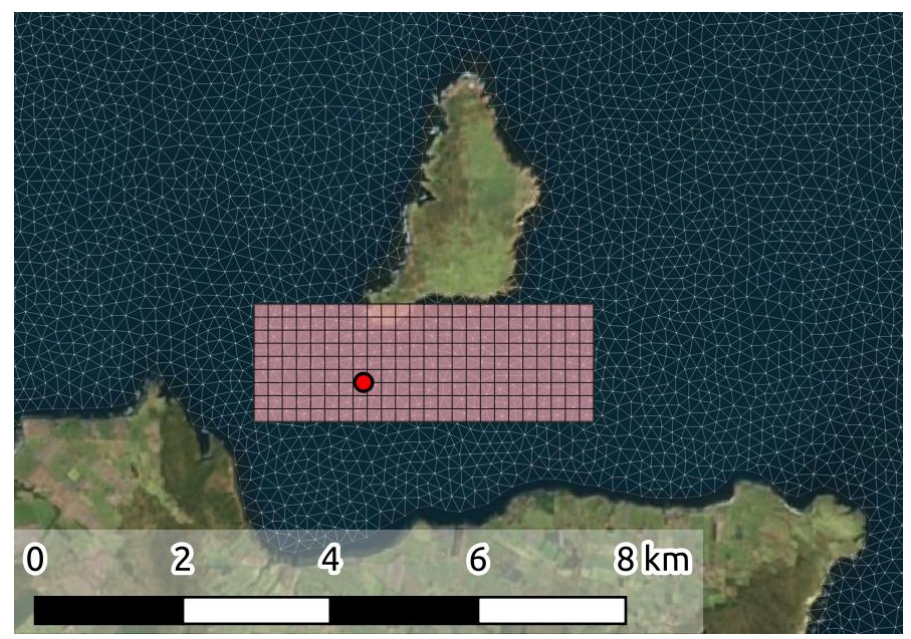

Figure 3: Red circle: location of ADCP used within this study. Polygon: grid of $24 \times 9$ cells; modelled velocities at cell centres are used to explore the spatial variation of non-deterministic flow features.

\section{Limits of predictability}

In this section, we present numerical experiments which reveal the presence of a non-deterministic component in the flow in the Inner Sound. We first present results from a model configuration forced at the boundary with the M2 harmonic constituent only, and make comparisons between each modelled tidal cycle. For a purely deterministic flow, the flow should be perfectly periodic with the M2 tidal period (12 h $25 \mathrm{~min}$ ). The variation in the flow field between tidal cycles can therefore be used as a proxy for the non-deterministic variability.

Figure 4 shows a timeseries of the modelled current at the ADCP location, from a simulation with $n=0.03 \mathrm{~s} \mathrm{~m}^{-1 / 3}$ and $\nu=1 \mathrm{~m}^{2} \mathrm{~s}^{-1}$, after a spin-up period of one month. The modelled flow does not tend towards a purely periodic solution; the peak velocity magnitudes within each tidal cycle (as marked in the figure) show high variability. As a proxy for this variability, we compute the standard deviation of the peak-to-peak current amplitudes within each tidal cycle. We compute this standard deviation over a simulated period of 36 days (or 69 M2 tidal cycles), after an initial spin-up period of five days. Figure 5 shows how this standard deviation depends on $n$ and $\nu$. We emphasise that for a fully deterministic flow, this standard deviation should be equal to zero, but that the presence of a nondeterministic component means that the flow field is inherently unpredictable, and therefore varies between each tidal period. The non-deterministic behaviour can be suppressed by increasing the viscosity $\nu$, or the friction coefficient $n$. Within the parameter ranges considered here, the viscosity has a stronger influence than the friction coefficient on the non-deterministic component of the flow.

Extending the above analysis to the 24 by 9 grid shown in figure 3, we obtain figure 6 , where the value for each cell is taken from its centre. This figure shows that the non-deterministic variability in the flow depends not only on the parameters $n$ and $\nu$, but also varies spatially across the Inner Sound. Note that, even for the most highly dissipative parameter values (i.e. for the plots towards the upper-right of figure 6), the relatively high variability to the south east of Stroma persists.

Instead of comparing different tidal cycles within a single model run, the non-deterministic effect can also be observed by generating an ensemble of model runs, and making direct comparisons between each ensemble member. Here, we generate such an ensemble by applying a small $\left(\mathcal{O}\left(10^{-4}\right) \mathrm{m} \mathrm{s}^{-1}\right)$ random perturbation to the velocity at a time 
$t=0$, following an initial spin-up period of five days. The perturbation is applied across the whole domain, and applied independently for each degree of freedom in the model velocity field to generate a spatially varying perturbation. We then make comparisons between the modelled flow fields at a later time, approximately five days after the perturbation is applied. This type of comparison facilitates the use of a full boundary condition (with 11 constituents), since it does not rely on assumptions about the periodicity of the underlying deterministic flow. Figure 7 shows a comparison between the vorticity fields from two ensemble members generated with $n=0.03 \mathrm{~s} \mathrm{~m}^{-1 / 3}$ and $\nu=1 \mathrm{~m}^{2} \mathrm{~s}^{-1}$. We find that the differences between the two simulations are substantial, and easily visible by eye. Figure 8 shows the results from an equivalent experiment, with $\nu=40 \mathrm{~m}^{2} \mathrm{~s}^{-1}$. In this higher-viscosity case, the differences between the two ensemble members are too small to see by eye. This is consistent with the results of figures 5 and 6 , where we find that a viscosity $\nu=40 \mathrm{~m}^{2} \mathrm{~s}^{-1}$ strongly suppresses the non-deterministic variability. However, comparing figures 7 and 8 , we see that much of the finer-scale flow structure is removed by the use of the higher viscosity value.

This loss of finer-scale structure can also be visualised by comparing the power spectral densities (PSD) for the modelled velocity at the ADCP location, for $\nu=1$ and $\nu=40 \mathrm{~m}^{2} \mathrm{~s}^{-1}$. This is shown in figure 9 for the $x$-component of the velocity, where we also plot the PSD of the ADCP data. The use of higher viscosity suppresses the PSD at all frequencies, but has a stronger effect on the noise between the peaks than on the peaks themselves. Furthermore, above a frequency of approximately five times the M2 harmonic frequency, the use of higher viscosity reveals peaks which could not be distinguished above the background noise for $\nu=1$, but which are present in the observation data.

Overall, the results of this section demonstrate the presence of a non-deterministic component in the flow within the Inner Sound, and highlight the trade-off between predictability (achieved by suppressing non-deterministic behaviour via high viscosity), and faithfully representing reality (e.g. for applications where the finer-scale eddy structure is important).

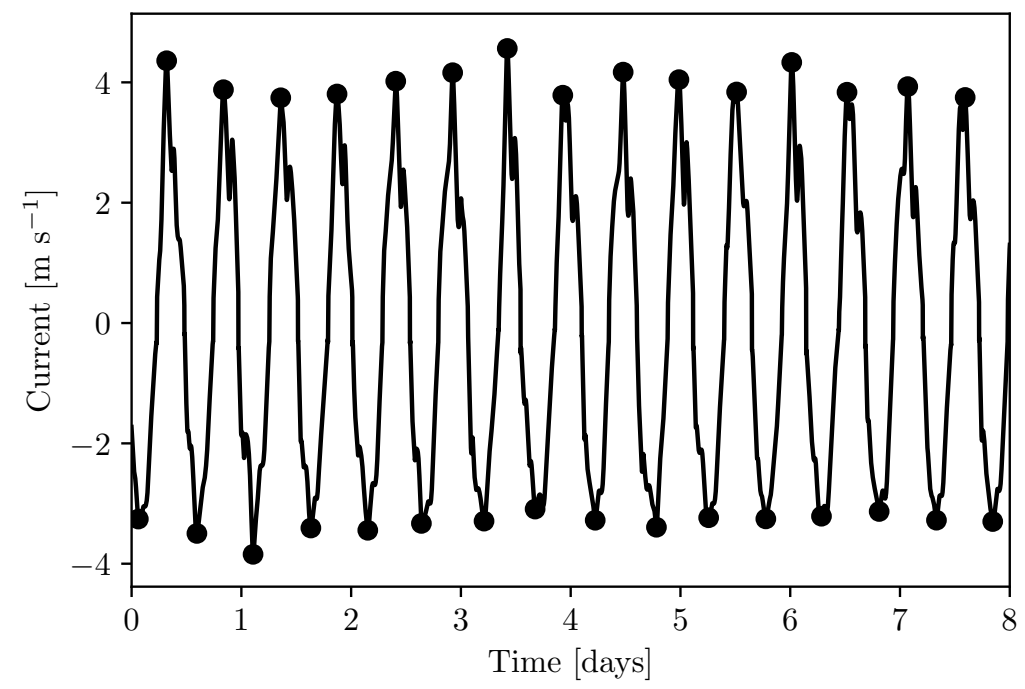

Figure 4: Modelled velocity at the ADCP location, for a model forced with the M2 constituent only. The model was run with $n=0.03$ $\mathrm{s} \mathrm{m}^{-1 / 3}, \nu=1 \mathrm{~m}^{2} \mathrm{~s}^{-1}$. The black circles indicate the peaks, whose variability can be used to quantify the non-deterministic effect. 


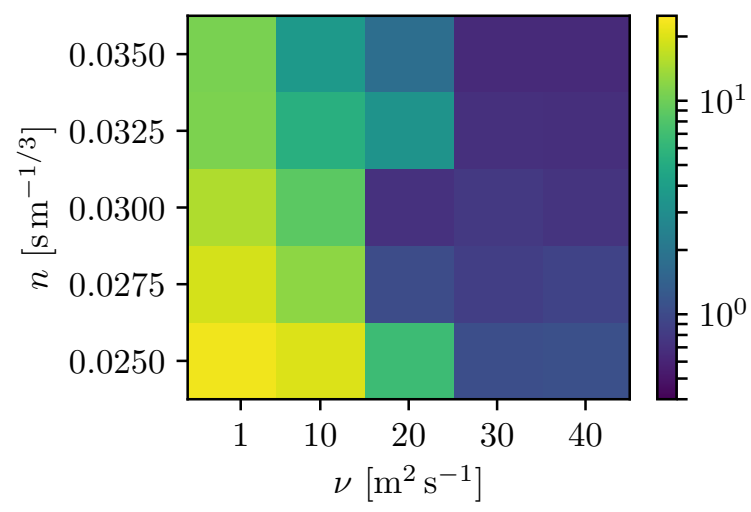

Figure 5: Standard deviation in modelled peak-to-peak current amplitude, over a 36-day simulation, for a model configuration forced with only the M2 constituent. Units: $\mathrm{cm} \mathrm{s}^{-1}$. Note the logarithmic scale. The variance should equal zero for a fully deterministic flow, but we find that the flow is non-deterministic, particularly for low $n$ and $\nu$.
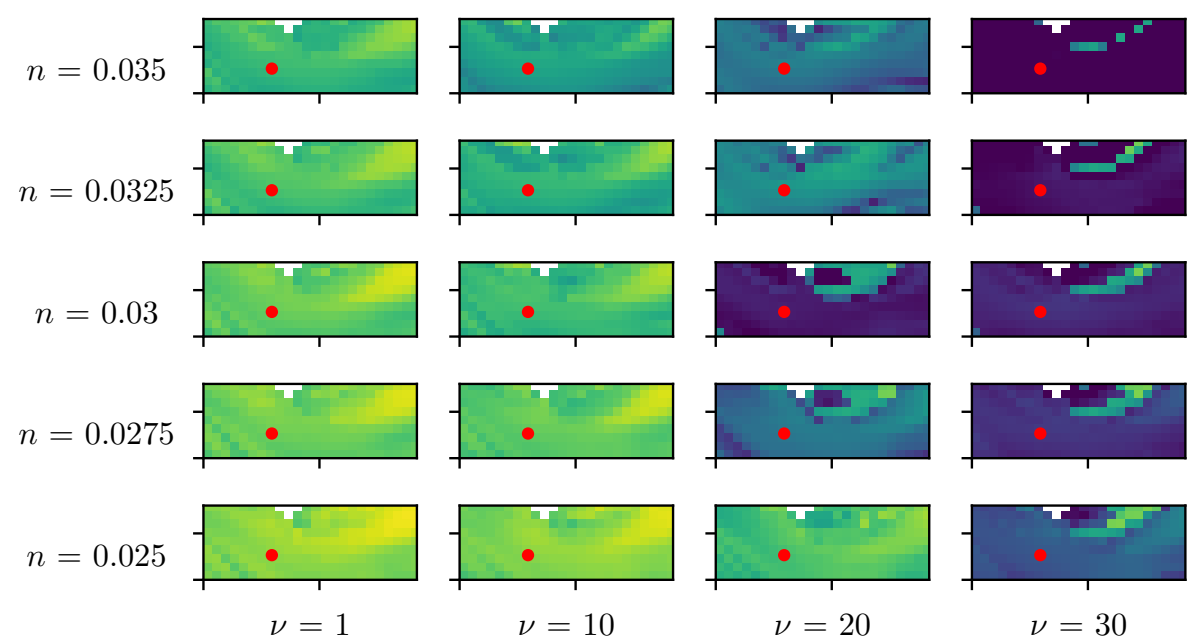

$\nu=10$

$\nu=20$
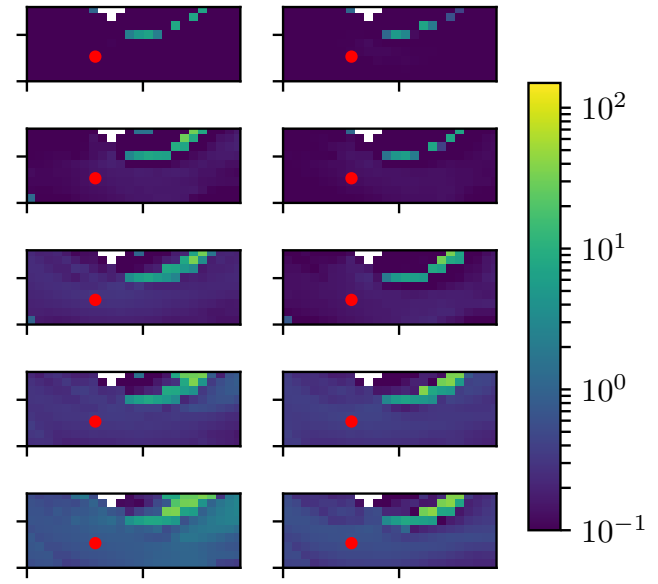

$\nu=30$

$\nu=40$

Figure 6: Standard deviation of peak-to-peak M2 current amplitude on the grid shown in figure 3, as a function of $n$ and $\nu$, based on a model forced with the M2 constituent only. Units: $\mathrm{cm} \mathrm{s}^{-1}$. This is an extension to figure 5 , which plotted this at the ADCP location only (indicated here by the red circle). This result demonstrates that the non-deterministic behaviour is not unique to the ADCP location, and that the magnitude of the non-deterministic component varies across the Inner Sound. 

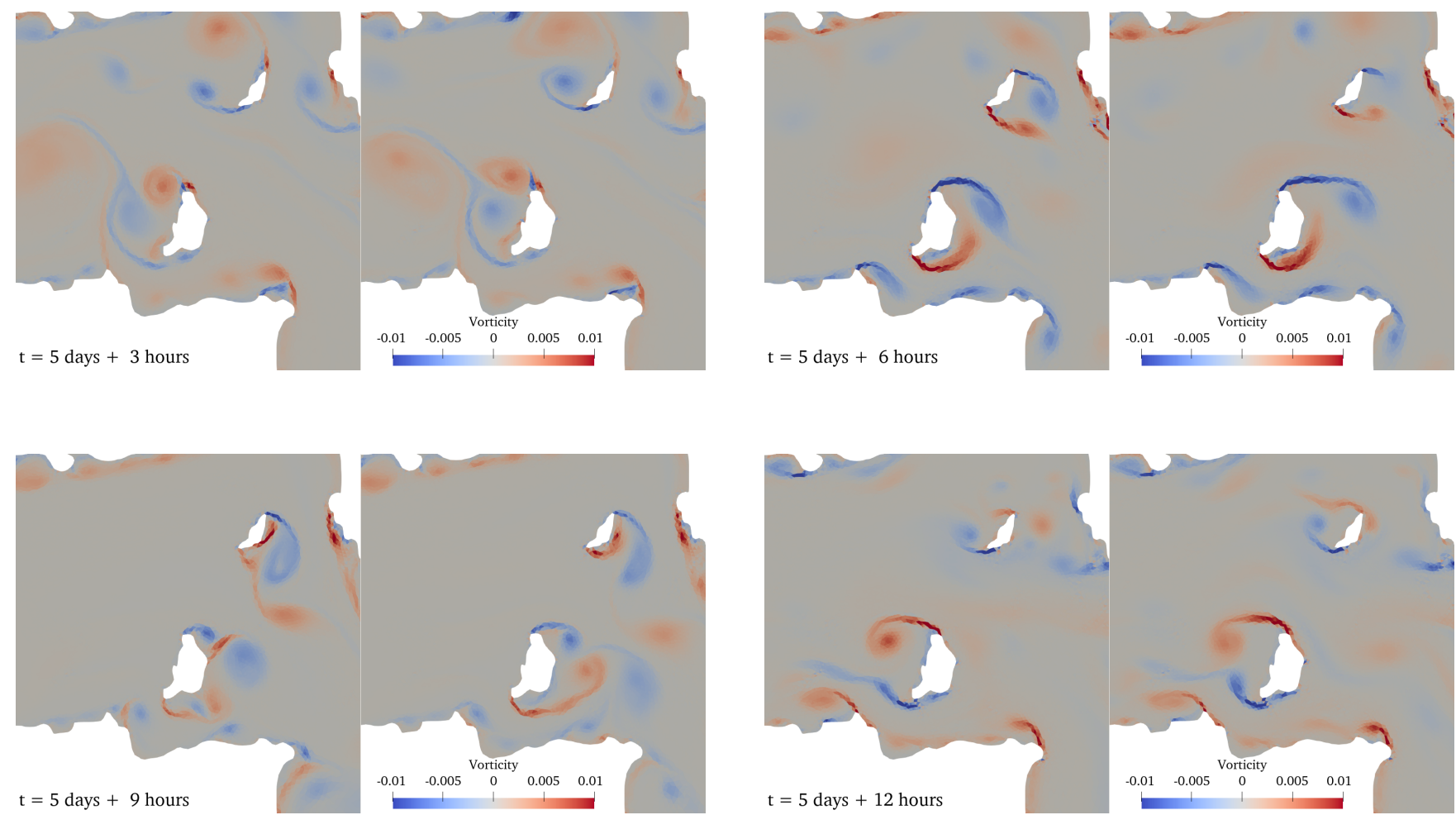

Figure 7: Modelled vorticity fields with $n=0.03 \mathrm{~s} \mathrm{~m}^{-1 / 3}, \nu=1 \mathrm{~m}^{2} \mathrm{~s}^{-1}$. Units: $\mathrm{s}^{-1}$. Each pair of plots is the vorticity field at the specified time, from a pair of simulations run with identical model parameters. The only difference between the two simulations is a small random perturbation on the order of $0.1 \mathrm{~mm} \mathrm{~s}^{-1}$ to the velocity field at time $t=0$.
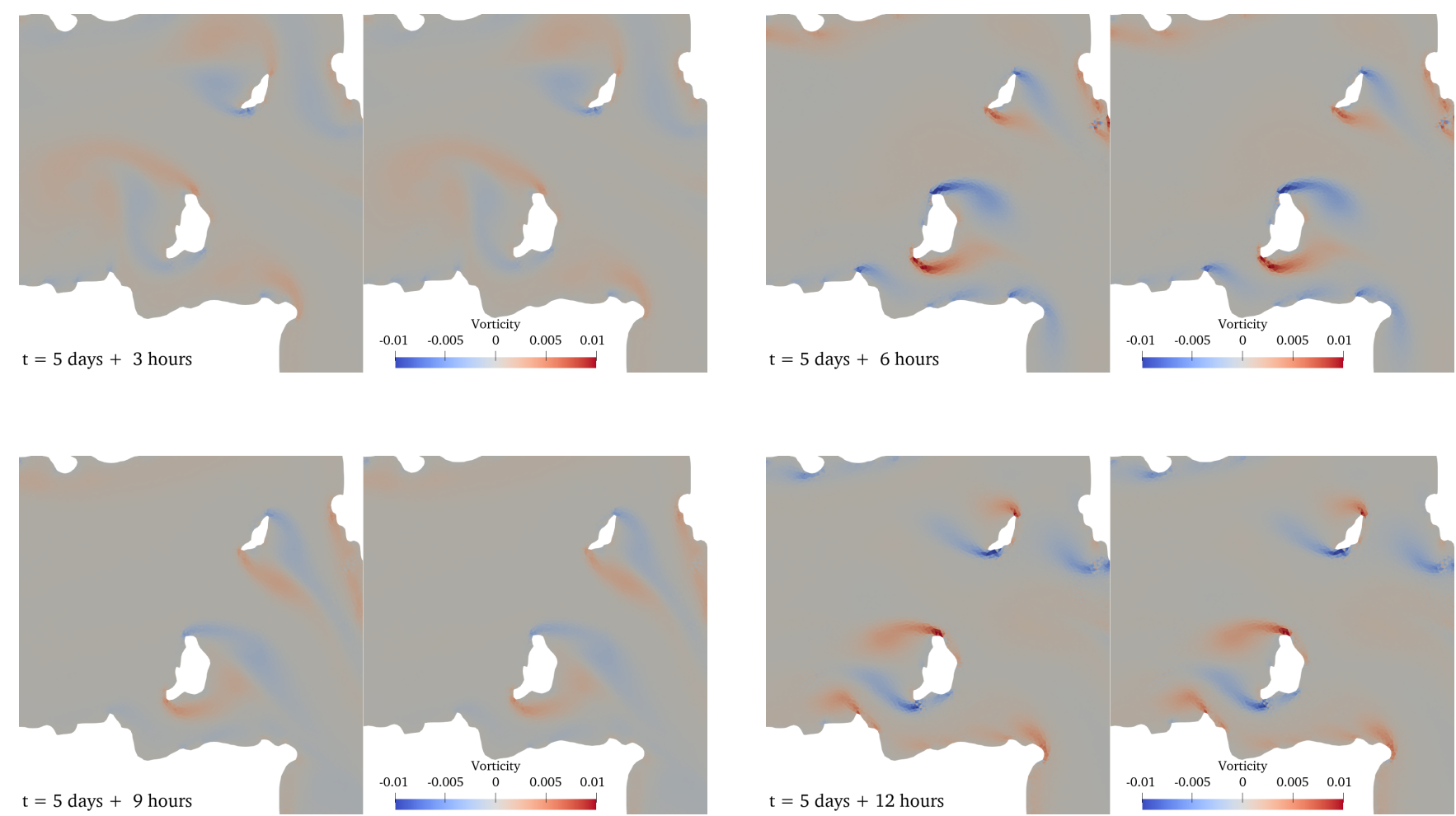

Figure 8: Equivalent results to figure 7, but with viscosity $\nu=40 \mathrm{~m}^{2} \mathrm{~s}^{-1}$. Units: $\mathrm{s}^{-1}$. The two perturbed simulations produce very similar fields, since the non-deterministic component of the flow is suppressed by the high viscosity. However, much of the complexity in the flow is lost. 


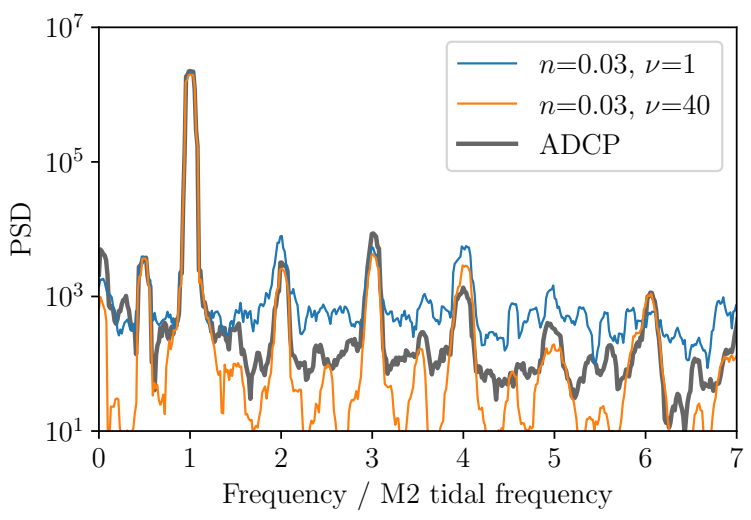

Figure 9: Power spectral density (PSD) of the $x$-component of the modelled velocity at the ADCP location, for two different values for $\nu$. This is consistent with the results from figures 7 and 8 , which show that the $\nu=1 \mathrm{~m}^{2} \mathrm{~s}^{-1}$ simulation contains a lot more fine-scale structure. However, this fine-scale structure contains a significant non-deterministic component, which can be suppressed by the higher viscosity parameter.

\section{Quantifying model performance}

As a preliminary step towards model calibration, in this section we explore a number of possible choices for error metrics based on comparisons with ADCP data. We can compare either raw velocity or, since the study region is of significant interest for its tidal stream energy potential, tidal stream kinetic power density (KPD, units $\mathrm{W} \mathrm{m}^{-2}$ ), given by

$$
\mathrm{KPD}=\frac{1}{2} \rho|\mathbf{u}|^{3}
$$

Based on either velocity or KPD, a large number of possible metrics exist by which to quantify the agreement between model outputs and observations. We consider the following quantities:

(i) Variance explained by the model, defined as $\operatorname{Var}\left(u_{\text {model }}\right) / \operatorname{Var}\left(u_{\text {obs }}\right)$. This is the ratio between the variance (over time) of the model outputs and the variance of the observations. A perfect model would have an explained variance of 1 .

(ii) Root mean squared error (RMSE), defined as $\sqrt{\text { mean }\left(u_{\text {model }}-u_{\text {obs }}\right)^{2}}$. A perfect model would have an RMSE of $0 \mathrm{~ms}^{-1}$.

(iii) Coefficient of determination, defined as $R^{2}=1-\operatorname{Var}\left(u_{\mathrm{obs}}-u_{\text {model }}\right) / \operatorname{Var}\left(u_{\mathrm{obs}}\right)$. A perfect model would have $R^{2}=1$.

The behaviour of these error metrics, applied to the modelled and observed velocity at the ADCP location, are shown in figure 10. We find that the RMSE and $R^{2}$ coefficient show very similar patterns, i.e. they are optimised by approximately the same values of $n$ and $\nu$. This is expected given their similar mathematical definitions. The explained variance, however, shows different behaviour. While the RMSE and $R^{2}$ coefficient appear to be optimised by approximately $n=0.03 \mathrm{~s} \mathrm{~m}^{-1 / 3}$ and $\nu=20 \mathrm{~m}^{2} \mathrm{~s}^{-1}$, the explained variance is optimised by smaller values for $n$, of around $0.0275 \mathrm{~s} \mathrm{~m}^{-1 / 3}$, and shows much smaller sensitivity to $\nu$. 
Figure 11 shows the same error metrics based on kinetic power density. The RMSE and $R^{2}$ plots are similar to those for the velocity-based error metrics, and are optimised by similar values of $n$ and $\nu$. The explained variance plot is again qualitatively similar to the equivalent velocity-based error metric, showing greater sensitivity to $n$ than $\nu$. However, in this case the explained variance is optimised by a slightly larger value for $n$, approximately $n=0.03$ $\mathrm{s} \mathrm{m}^{-1 / 3}$. In general, the explained variance ratios are higher for modelled KPD than for modelled velocity, hence the need for a larger $n$ to compensate. This is because the modelled velocities tend to exhibit excess variance when the currents are highest, while tending to under-predict the true variance at lower velocities. Since the KPD is related to the cube of the velocity, higher velocities have a greater weight in the KPD. It follows that the explained variances for the KPD are are greater than those for the velocity.

We can additionally compute error metrics based on a harmonic analysis of the velocity. For this, we focus on the $x$-component of the velocity, since harmonic analysis cannot be applied to the combined velocity given by equation (3). We perform harmonic analysis on both the model outputs and the observation data, over the full 33-day ADCP deployment period. Under the constraints of the Rayleigh criterion, this time period permits an analysis for the five largest tidal constituents present in the ADCP signal (M2, S2, N2, $\mu 2$, M4). A combined error metric for comparing the modelled and observed amplitude and phase of these constituents is given by

$$
\mathrm{RMSE}_{\text {harmonic }}=\sqrt{\sum_{C} \frac{1}{2}\left(A_{C}^{2}+\hat{A}_{C}^{2}\right)-A_{C} \hat{A}_{C} \cos \left(\phi_{C}-\hat{\phi}_{C}\right)}
$$

where $A_{C}$ and $\hat{A_{C}}$ are the modelled and observed amplitudes of the constituent $C$, respectively, and $\phi_{C}$ and $\hat{\phi}_{C}$ are the corresponding phases (Cummins and Oey, 1997). The value of this error metric as a function of $n$ and $\nu$ is shown in figure 12. We find that this error metric has a well-defined optimum, at approximately $n=0.03 \mathrm{~s} \mathrm{~m}^{-1 / 3}, \nu=1$ $\mathrm{m}^{2} \mathrm{~s}^{-1}$.

We can also use this harmonic analysis to compare the explained variance of the harmonic residual of the velocity. Defined as the difference between the full velocity signal and the fit based on harmonic analysis, the harmonic residual contains several components:

(i) Harmonic constituents not included within the harmonic analysis. This is inevitable, since the ADCP data spans a limited time period, and the set of constituents which can be resolved by harmonic analysis is limited by the Rayleigh criterion.

(ii) Eddies and similar geometry-induced flow structures occurring at non-harmonic frequencies. Such features have a significant presence in and around the Inner Sound, as demonstrated in figure 7.

(iii) Smaller scale circulation/turbulence, although this should be somewhat reduced by the 10-minute time-averaging of the observation data and model outputs.

(iv) Perturbations due to weather events e.g. storm surge, which we assume are small.

The variance of the non-harmonic component is, by definition, relatively more sensitive to the non-deterministic component than the variance of the full flow. While it is not possible to achieve an exact match between the modelled and observed non-deterministic flow, there may exist a model configuration in which the statistics of the non-deterministic dynamics are in some way representative of the observed flow. As such a statistic, we choose to use the explained 
variance of the non-harmonic component; that is, the variance of the modelled non-harmonic component, normalised by the variance of the observed non-harmonic component. This is shown in figure 13 as a function of the model parameters. A model configuration capturing the true non-harmonic variability in the flow would have a value of unity for this error metric. Within the parameter ranges plotted, $n$ and $\nu$ have very similar influence on the non-harmonic variance. This is in contrast to the explained variance of the full flow, which shows less sensitivity to $\nu$. This is consistent with the results of section 3, where we found that the influence of the viscosity is stronger on the non-deterministic component than on the underlying deterministic flow.

Finally, we can also consider the ratio of the modelled to observed mean kinetic power density, over the ADCP deployment period. This is shown in figure 14. The optimal value of this metric is 1 , which can be achieved within the model by using a value of $n$ around 0.0275 , and shows little sensitivity to $\nu$. Note that the use of the mean KPD allows for periods of over- and under-estimation to cancel out, which is not the case for error metrics based on variance or RMSE. The viscosity sensitivity shown by the KPD RMSE and $R^{2}$ error metrics indicates that the viscosity does influence the KPD (and hence also the forces on turbines, etc) on short time scales. However, for many resource assessment studies, the mean KPD may be the model output of interest, and these results indicate that this error metric has limited sensitivity to the model viscosity.

The above error metrics fall into four categories, with different characteristic patterns when plotted in $n-\nu$ space:

1. The explained variance of both the velocity and KPD, and the modelled mean KPD, all show greater sensitivity with respect to $n$ than with respect to $\nu$, within the parameter ranges explored.

2. The RMSE and $R^{2}$ coefficient of both the velocities and kinetic power densities have reasonably well-defined optimal points within the parameter space (the optimum is a point rather than a line), and all four of these metrics are optimised by similar values of $n$ and $\nu$. Note, however, that performing model calibration to optimise these error metrics might artificially favour high viscosity or friction coefficient; this issue is discussed in greater detail in section 7 .

3. The harmonic analysis-based RMSE identifies an optimal pair of model parameters at around $n=0.03 \mathrm{~s} \mathrm{~m}^{-1 / 3}$, $\nu=1 \mathrm{~m}^{2} \mathrm{~s}^{-1}$.

4. The explained variance of the harmonic residual of the velocity, as shown in figure 13, shows similar sensitivity to both $n$ and $\nu$.

While it is not possible to simultaneously achieve model-observation agreement in all error metrics, it is possible to achieve certain combinations. For example, the pattern exhibited by the harmonic residual variance (figure 13) is unique, and model-observation agreement can be achieved in combination with any error metric from group 1 above. Meanwhile, groups 2 and 3 above each identify optimal combinations of $n$ and $\nu$. This presents a variety of options for proceeding with model calibration efforts. 

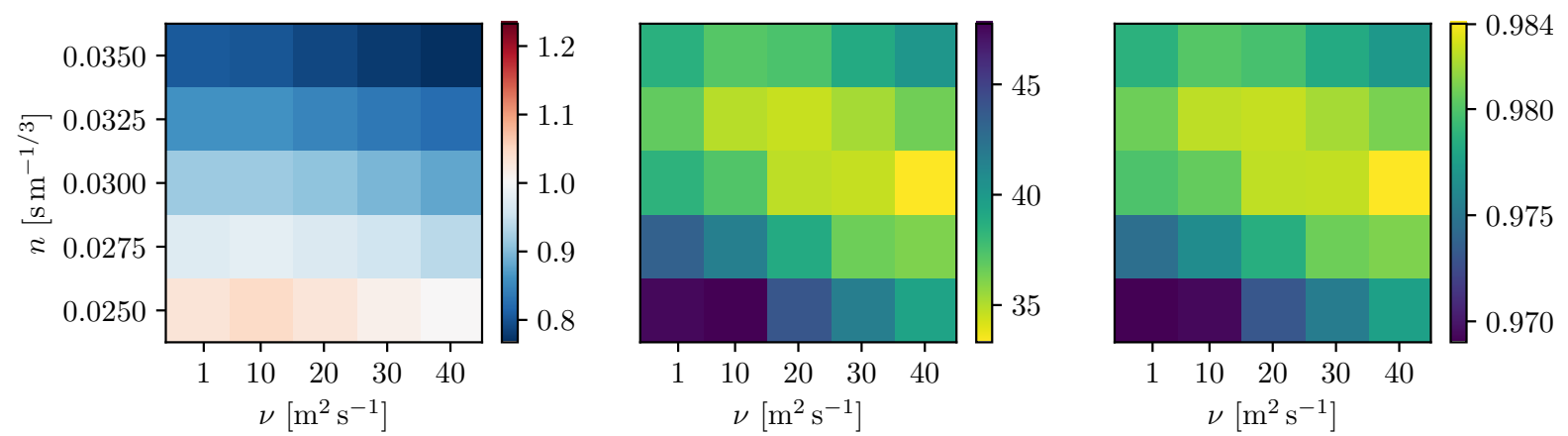

Figure 10: Velocity error metrics, as a function of $n$ and $\nu$. Left: explained variance. Middle: RMSE [cm]. Right: $\mathrm{R}^{2}$ coefficient.
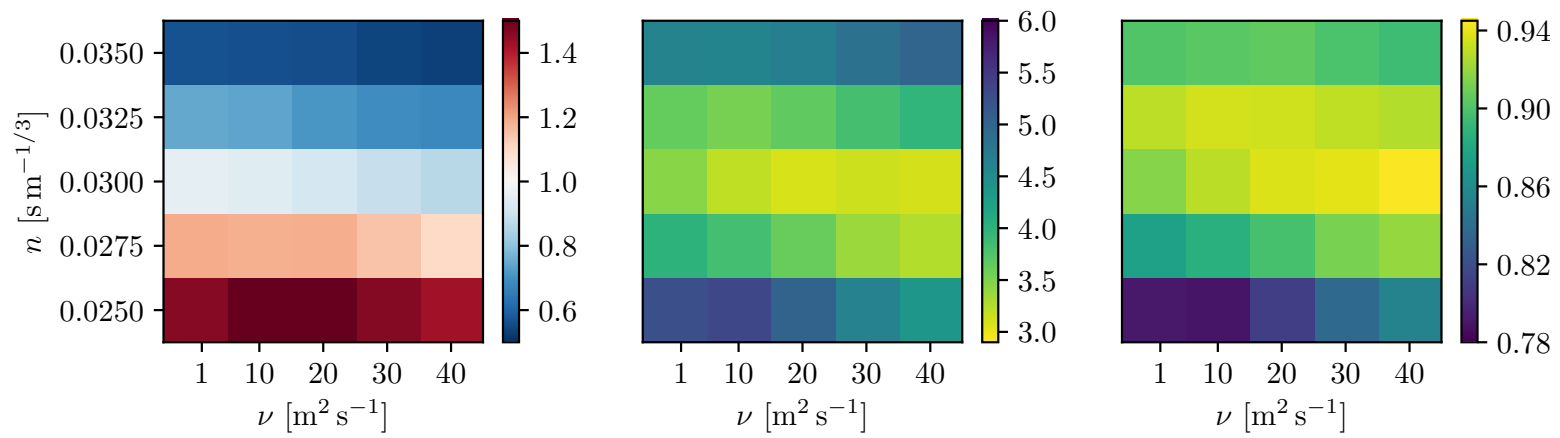

Figure 11: Kinetic power density error metrics, as a function of $n$ and $\nu$. Left: explained variance. Middle: RMSE [kW $\mathrm{m}^{-2}$ ]. Right: $\mathrm{R}^{2}$ coefficient.

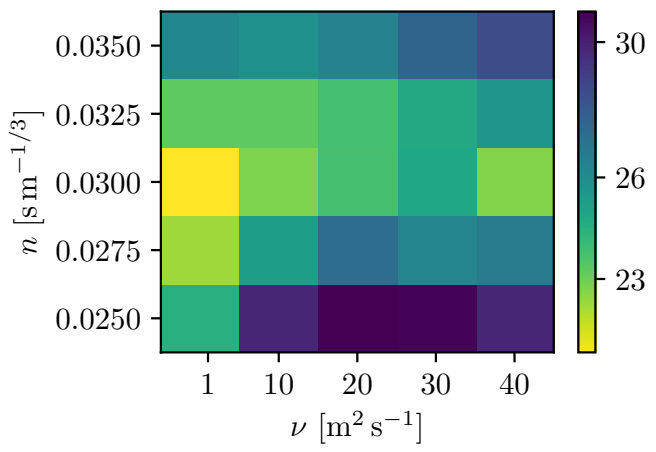

Figure 12: The harmonic analysis-based RMSE (given by equation (5)), as a function of $n$ and $\nu$. Units: $\mathrm{cm} \mathrm{s}^{-1}$. 


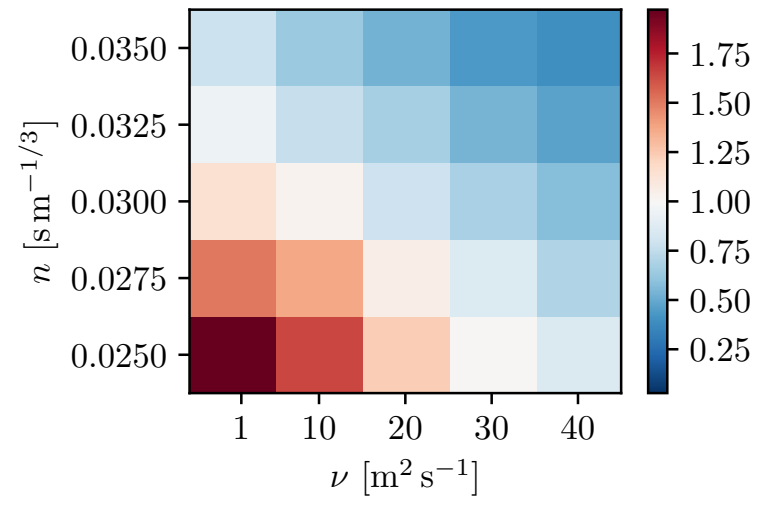

Figure 13: The harmonic residual variance of the model (as a function of $n$ and $\nu$ ), divided by the harmonic residual variance of the ADCP observation data. Where this ratio is equal to 1 , the non-harmonic variability of the model matches that of the observations.

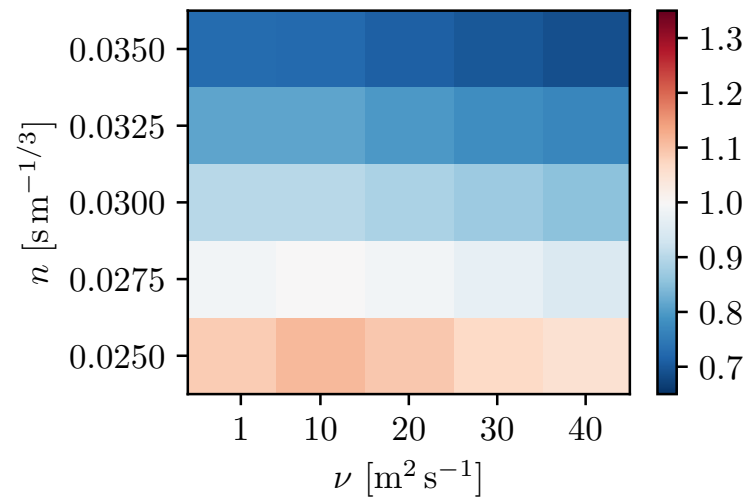

Figure 14: Ratio of modelled to observed mean kinetic power density at the ADCP location, as a function of $n$ and $\nu$.

\section{Towards model calibration, in the presence of non-deterministic effects}

The results of section 4 show that all of the considered error metrics appear to vary smoothly over the parameter range, when plotted at a coarse parameter resolution. The results are therefore sufficient for a crude model calibration exercise; values for $n$ and $\nu$ can be selected which approximately optimise any given choice of error metric. However, depending on the magnitude of the non-deterministic component of the flow, there will be a fundamental lower limit to the optimal parameter resolution achievable. That is, for sufficiently small perturbations to $n$ and $\nu$, the influence of the perturbation will be indistinguishable above the 'noise' introduced by the non-deterministic effect. As a result, the error metrics will not vary smoothly with the input parameters, preventing the precise identification of optimal parameters and potentially limiting the choice of possible calibration methods. In this section, we investigate this lower limit on achievable parameter resolution, focusing on the friction parameter, $n$.

Figure 15 shows the perturbations in the modelled mean KPD over a 10-day period resulting from perturbations in the Manning coefficient of varying sizes. For these small perturbations, we would expect to see a linear relationship between the input and output perturbations. However, due to the non-deterministic component (whose size varies with the viscosity $\nu$ ), there exists a viscosity-dependent threshold below which model-observation comparisons cannot distinguish between Manning coefficients. That is, for a sufficiently small perturbation in the Manning coefficient, the resulting perturbation in the model output (or error metric) is lost within the uncertainty introduced by the nondeterministic effect. In order to sample the non-deterministic variability, here we have generated ensembles of 12 model runs with randomly perturbed initial conditions. We then estimate the non-deterministic uncertainty as two times the standard deviation of the error metric over the ensemble. This uncertainty is indicated by the horizontal lines in figure 15. The intersection of each horizontal line with the 'linear' relationship maps this non-deterministic uncertainty onto a threshold value for $\Delta n$. For perturbations smaller than this threshold value, we observe that the output perturbation is no longer linearly proportional to the input perturbation, for the reasons described above. Specifically, we find that for a model configured with $\nu=1 \mathrm{~m}^{2} \mathrm{~s}^{-1}$, it is not possible to distinguish between Manning coefficients which differ 
by much less than $\Delta n=10^{-3} \mathrm{~s} \mathrm{~m}^{-1 / 3}$ using this error metric. For $\nu=40 \mathrm{~m}^{2} \mathrm{~s}^{-1}$, this threshold decreases to less than $\Delta n=10^{-7} \mathrm{~s} \mathrm{~m}^{-1 / 3}$.

However, as well as depending on $\nu$, these parameter identifiability thresholds also depend on the selected error metric. Figure 16 compares the thresholds for a variety of error metric choices, in combination with different viscosity values. The mean KPD is the least affected by the non-deterministic behaviour. This is likely to be because the time-averaging allows periods of under- and over-estimation to cancel out over time. The error metric based on the velocity harmonic analysis (given by equation (5)) also performs well at low viscosity, because the harmonic analysis acts to filter out the high-frequency variability from the signal. The $R^{2}$ and RMSE measures based on the KPD perform the worst. However, the choice of viscosity has a far greater effect than the choice of error metric.

The thresholds also depend on the time range over which the error metric is computed. Given a stationary underlying flow, the standard deviations over the non-deterministic ensemble should reduce smoothly as the error metric analysis period increases. However, due to the periodicity in the underlying flow on the scale of weeks (the spring-neap cycle) and hours (individual tidal cycles), the behaviour of the thresholds with respect to time is not smooth. Nonetheless, we found through additional experiments (not shown) that, for any analysis period longer than a few days, the choice of error metric and viscosity are more influential than the length of the analysis period in determining the achievable parameter resolution. In practice, performing model-observation comparisons over the full time range of available data is always preferable, although this may not always be possible depending on the choice of calibration method and the available computational resources (this is discussed in section 7).

In summary, the viscosity, choice of error metric and model-observation comparison duration are all factors in determining the influence of the non-deterministic effect within a calibration problem for the Manning coefficient $n$. In particular, these factors dictate the minimum perturbation in the Manning coefficient capable of producing output perturbations which can be resolved above the uncertainty introduced by the non-deterministic component of the flow. We have found that the mean kinetic power density is the most robust error metric with respect to the non-deterministic effects.

\section{Effect of mesh resolution}

In this section we briefly explore the effect of mesh resolution on a selection of error metrics. The meshes differ only in their refinement in and around the Inner Sound, as shown in figure 1. The primary mesh used for all of the above results uses a uniform $200 \mathrm{~m}$ resolution within the Pentland Firth. The two refined meshes employ $100 \mathrm{~m}$ and $50 \mathrm{~m}$ resolution within the Inner Sound.

Figures 17, 18, 19 and 20 compare model performance across these meshes, based on the mean KPD, KPD RMSE, velocity RMSE and harmonic analysis error metrics, respectively. In each case, the variation of the error metric with the parameters $n$ and $\nu$ is similar across all mesh resolutions. All meshes favour similar values for the parameters,

but overall model accuracy improves as mesh resolution is enhanced. These results suggest that calibrated parameters may be applicable across meshes of different resolutions. Since coarse meshes are less demanding on computational resources, this suggests that it may be beneficial to perform preliminary model calibration on a coarse mesh, before further tuning on a finer mesh. 


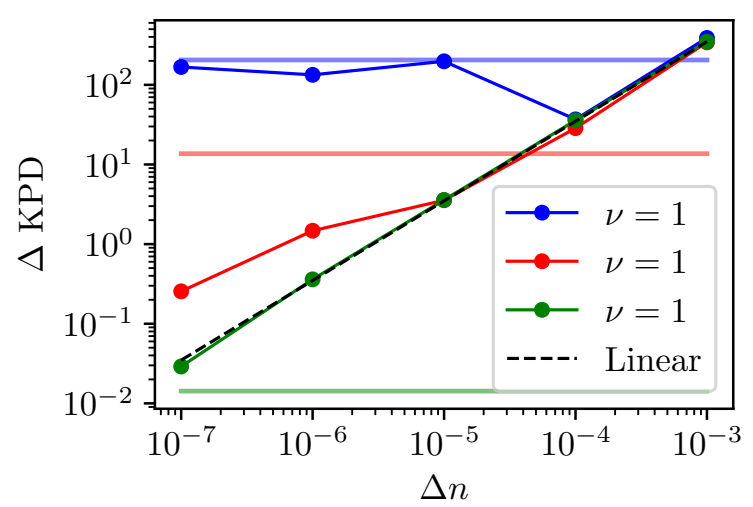

Figure 15: Perturbations $\triangle \mathrm{KPD}$ (units $\mathrm{W} \mathrm{m}^{-2}$ ) in modelled mean kinetic power density over a ten-day averaging period, resulting from perturbations $\Delta n\left(\mathrm{~s} \mathrm{~m}^{-1 / 3}\right)$ in the Manning coefficient, for a variety of different viscosity values $\nu\left(\mathrm{m}^{2} \mathrm{~s}^{-1}\right)$. For small perturbations, we expect to see a linear relationship between the Manning coefficient perturbation and the modelled mean power density, as indicated. The horizontal lines indicate two times the standard deviation of the mean KPD over an ensemble of randomly perturbed model runs, and are an estimate of the non-deterministic variability of the model result. We see that the output perturbations depart from the linear relationship once they drop below the corresponding threshold.

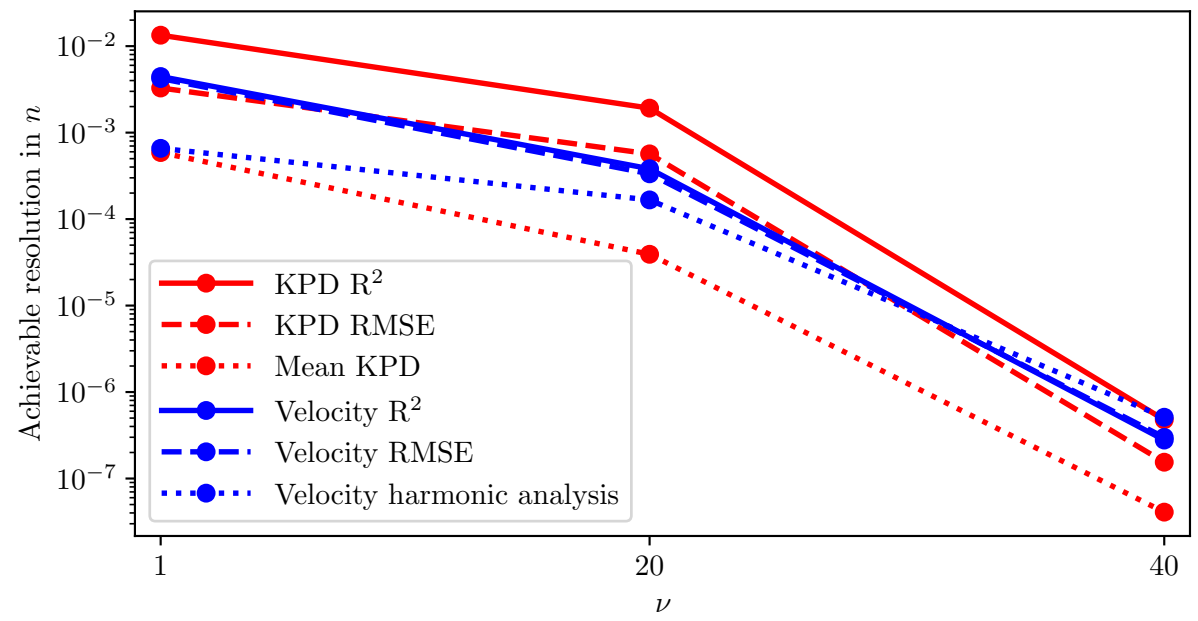

Figure 16: Comparison of achievable optimal parameter resolution based on each choice of error metric.
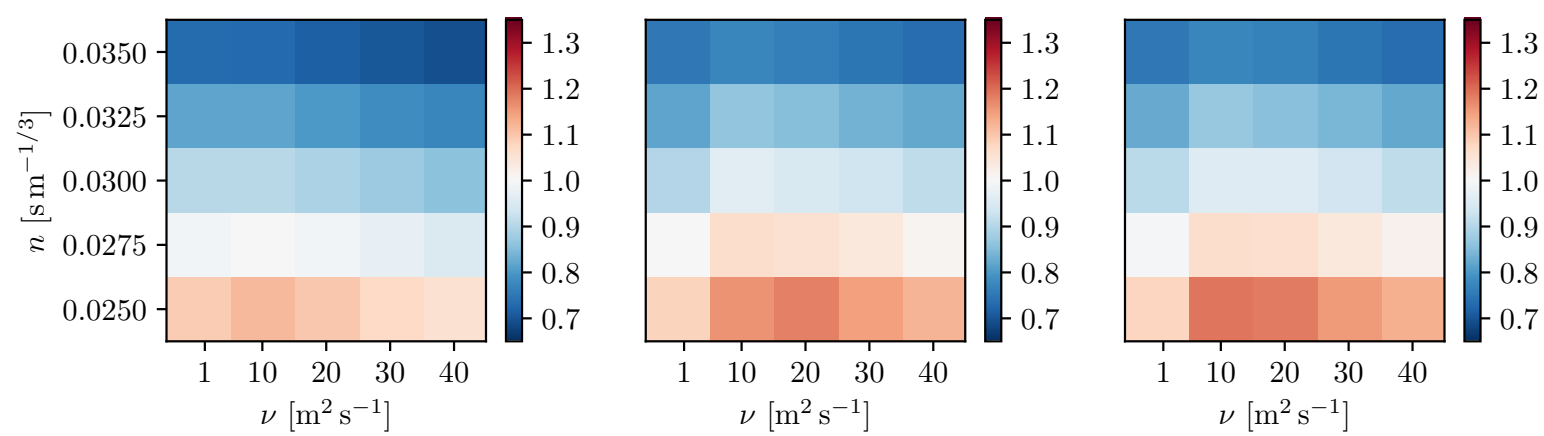

Figure 17: Ratio of modelled to observed mean kpd, as a function of $n$ and $\nu$, for various mesh resolutions. Left: 200m inside Inner Sound. Middle: 100m inside Inner Sound. Right: 50m resolution inside Inner Sound. The results are similar across all meshes. 

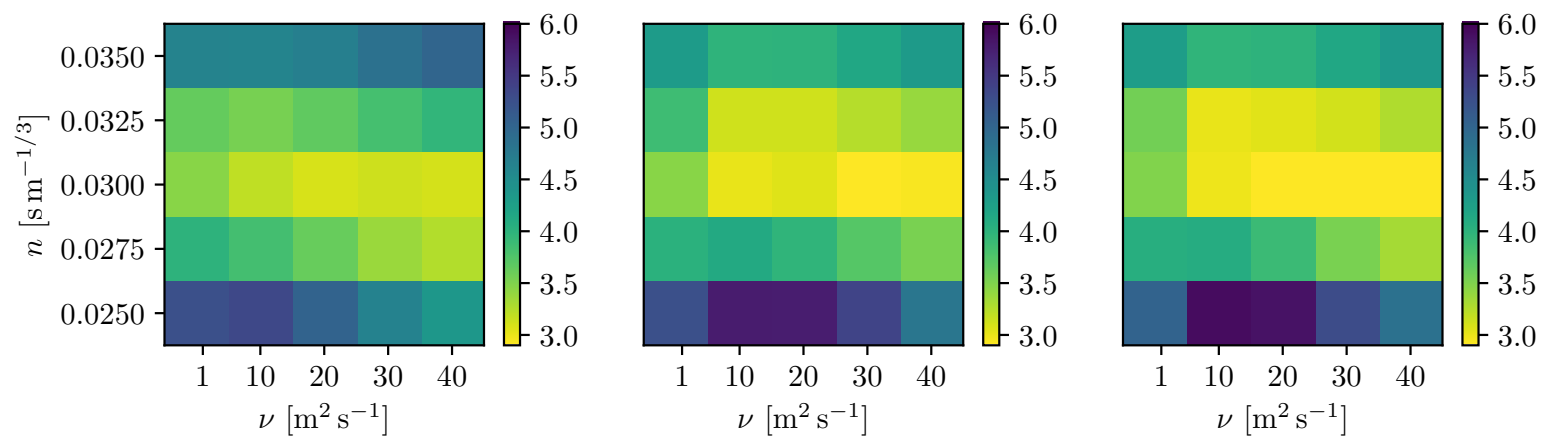

Figure 18: RMSE of modelled KPD, as a function of $n$ and $\nu$, for various mesh resolutions. Units: $\mathrm{kW} \mathrm{m}^{-2}$. Left: $200 \mathrm{~m}$ inside Inner Sound. Middle: 100m inside Inner Sound. Right: 50m resolution inside Inner Sound. The patterns for each mesh are very similar, but the RMSE values tend to decrease with finer mesh resolution.
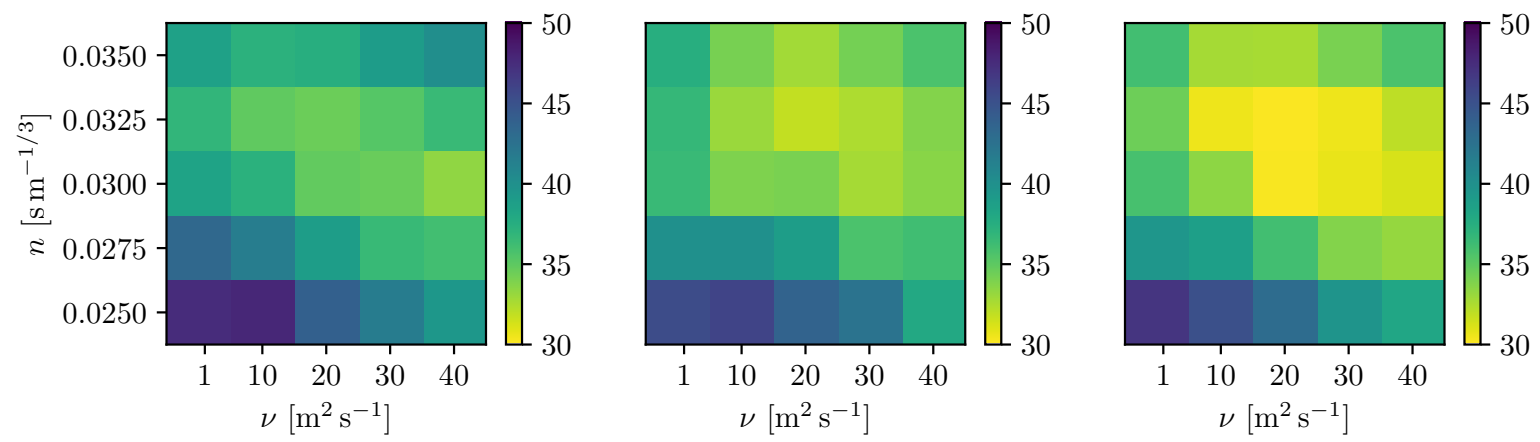

Figure 19: RMSE of modelled velocity, as a function of $n$ and $\nu$, for various mesh resolutions. Units: $\mathrm{cms}^{-1}$. Left: $200 \mathrm{~m}$ inside Inner Sound. Middle: 100m inside Inner Sound. Right: 50m resolution inside Inner Sound. Similarly to the KPD RMSEs of figure 18, the patterns for each mesh are very similar, although model performance improves with mesh refinement.
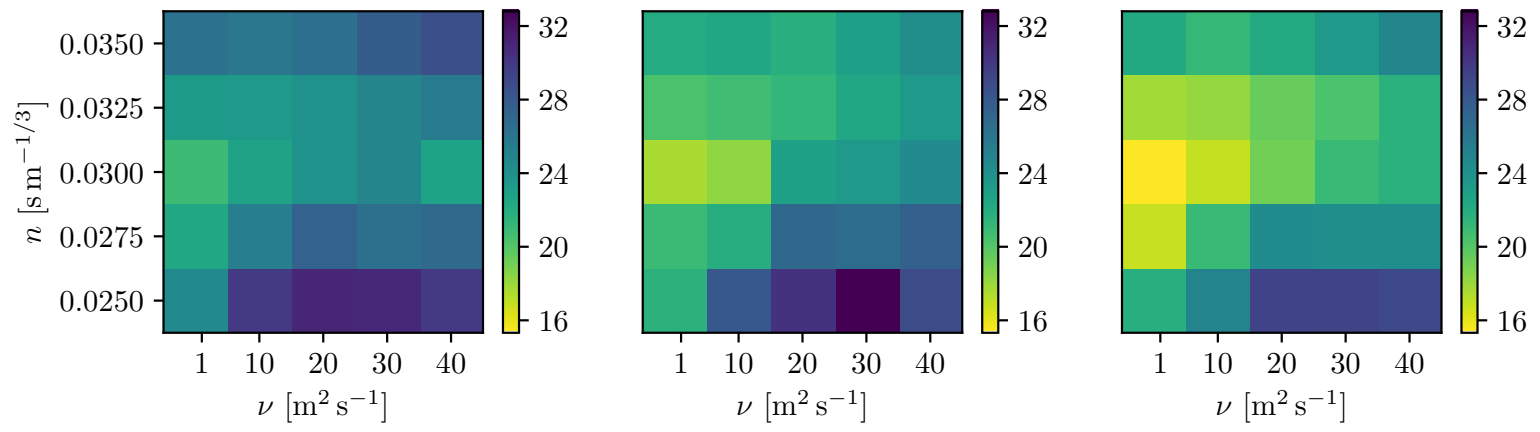

Figure 20: Harmonic analysis-based RMSE, as a function of $n$ and $\nu$, for various mesh resolutions. Units: $\mathrm{cm} \mathrm{s}^{-1}$. Left: $200 \mathrm{~m}$ inside Inner Sound. Middle: 100m inside Inner Sound. Right: $50 \mathrm{~m}$ resolution inside Inner Sound. The pattern is again similar for each mesh, with the lowest RMSEs achieved on the finest resolution mesh. 


\section{Discussion}

The results presented in section 3 demonstrate the presence of a non-deterministic component within the flow through the Inner Sound. This has significance for a number of reasons:

(i) There is a fundamental limit to the predictability of the flow, and hence the tidal stream resource, in the Inner Sound.

(ii) Non-deterministic flow implies non-harmonic flow, and harmonic analysis is therefore insufficient for predicting the flow within the Inner Sound.

(iii) Numerical models can capture the non-harmonic dynamics, but cannot achieve an exact match with observations. However, through model tuning, it is possible to achieve agreement in the statistics of the modelled and observed non-harmonic component.

(iv) Uncertainty is introduced into the model calibration problem, placing a fundamental limit on achievable parameter precision.

The results of sections 4 and 5 demonstrate that, while a simple model calibration is possible at a coarse parameter scale, caution must be exercised. In particular, we have shown that the uncertainty introduced by the non-deterministic behaviour places a limit on the parameter resolution which can be achieved via model-observation comparisons. Note that this initial model calibration has been based on a spatially uniform friction coefficient applied over the whole domain. In practice, a typical model calibration study would seek to estimate a spatially varying parameter, and the parameter identifiability limit would depend on both the number of parameters being estimated, and the volume of observation data available (Warder and Piggott, 2021). This is because, while the uncertainty introduced by the non-deterministic behaviour is constant, the influence of each friction parameter varies with the spatial region over which it is applied. An attempt to estimate multiple friction parameters corresponding to different sections of the domain would exacerbate the identifiability issue.

Figure 16 shows that the mean KPD is more robust than the RMSE- or $R^{2}$-based error metrics, with respect to the non-deterministic behaviour. This can be explained by the fact that periods of under- or over-estimation of the KPD are allowed to cancel out. From the perspective of tidal stream resource assessment, this is also the most relevant model output, and the priority for accurate modelling. Another advantage of calibrating based on mean KPD is that this metric shows relatively low sensitivity to the viscosity, as shown in figure 14. This suggests the possibility of a two-step calibration process. Calibration with respect to the friction coefficient can be performed at high viscosity (and hence potentially lower mesh resolution), which further increases robustness with respect to the non-deterministic behaviour. Once calibrated at high viscosity, the viscosity can then be reduced in order to achieve good performance on a second error metric, for example to capture the correct variance of the non-harmonic component in the flow, or to minimise the harmonic analysis-based RMSE. The explained variance of the velocity or KPD also exhibits similarly low sensitivity to the viscosity, so could be treated in a similar way to the mean KPD, by first calibrating for $n$ at high viscosity, then subsequently reducing viscosity as required.

The RMSEs and $R^{2}$ coefficients for the velocity and KPD (figures 10 and 11) show a different pattern of sensitivity, implying the existence of optimal values for both $n$ and $\nu$. In particular, these error metrics favour relatively high 
values of the viscosity (around $\nu=20-30 \mathrm{~m}^{2} \mathrm{~s}^{-1}$ ). However, in the presence of high variability on short time scales, this apparent preference for high viscosity could be misleading. This is because the RMSE between two noisy signals with the same underlying statistics (e.g. the ADCP data and a well-calibrated low-viscosity model run) is greater than that between a noisy signal (the ADCP data) and a smooth signal with the same underlying mean, but reduced variance (e.g. a high-viscosity model run). This agreement is spurious because in this scenario, the low-viscosity model is actually the better representation of reality, despite returning a higher RMSE. For this reason, we suggest caution in analysing model performance based only on RMSE- and $R^{2}$-based error metrics, and that other error metrics such as those based on harmonic analysis, mean KPD or explained variance (of the whole flow or only the non-harmonic part) should be considered.

In section 6 we compared model performance on a variety of meshes, whose resolution differ within the Inner Sound. The model performance improved as the mesh was refined within the Inner Sound. However, the optimal parameters remained approximately consistent across different mesh resolutions, suggesting that parameters obtained via a preliminary model calibration at coarse resolution can subsequently be applied to a higher-resolution model, possibly with further fine-scale tuning. Since the viscosity term represents a subgrid-scale process, the viscosity parameter is in principle mesh resolution-dependent, typically varying with the square of the grid size (Jones et al., 2007). The proposed use of high viscosity to suppress non-deterministic behaviour might therefore be consistent with the use of coarse model resolution, which will save computational cost within a preliminary calibration phase.

The results of this study also have implications for the selection of a suitable model calibration method. Many model calibration studies employ adjoint methods, whereby the gradient of some misfit functional with respect to the (spatially varying) model parameters can be computed at low computational cost. This enables the use of gradientbased optimisation methods for determining the optimal parameters (Heemink et al., 2002, Lu and Zhang, 2006, Chen et al., 2014). However, the presence of non-deterministic behaviour means that the misfit functional does not vary smoothly with respect to the control parameter (typically the field of friction coefficient values). This poses a barrier to gradient-based methods, since the misfit functional will not be convex with respect to the control parameters, and the presence of local minima is therefore likely to prohibit convergence to the global minimum. Here, the use of coarse resolution in conjunction with high viscosity (as suggested above) may be beneficial for three reasons. Firstly, the smoothness of the error metrics as a function of the friction parameter is improved through the use of a high viscosity value. Secondly, the use of coarse resolution reduces the computational demand. Adjoint methods require that the full model solution at all timesteps is stored in memory (or the use of a suitable checkpointing scheme), which can be prohibitive when the evaluation of the misfit functional requires long simulations. The use of coarse resolution reduces this memory demand. Thirdly, the suppression of non-deterministic behaviour reduces the time period over which the misfit need be computed, further reducing the memory demand. These advantages may also apply to alternative model calibration approaches.

Finally, we note that the findings of this study are unlikely to be unique to the Inner Sound of the Pentland Firth as studied within this work. The presence of a non-deterministic flow component is likely to affect any high-energy site with complex bathymetry and/or coastline geometry, and the results of this study generalise to any such site.

With these recommendations in hand, in future work we will perform a more extensive model calibration, using further sources of observation data. The use of additional data will facilitate the estimation of a spatially varying 
friction parameter, resulting in a robustly calibrated model suitable for tidal stream resource assessment.

\section{Conclusion}

Through numerical experiments, we have demonstrated the presence of a significant non-deterministic component within the flow through the Inner Sound of the Pentland Firth, a highly energetic site between mainland Scotland and the Orkneys which is of particular interest for its high tidal stream energy resource. This non-deterministic behaviour can be identified either by forcing the model with a single tidal constituent and inspecting the departure from periodicity in the resulting flow, or by comparing two or more model runs with perturbed initial conditions. This behaviour suggests a fundamental limit to the predictability of the flow in the Inner Sound, which has implications for tidal stream resource assessment, and also introduces additional uncertainty into efforts to calibrate the model. Furthermore, we expect that this issue is not unique to the Inner Sound of the Pentland Firth as studied here, and will be present in any other complex, high-energy site.

In order to calibrate a numerical model with respect to its bottom friction parameter, it is necessary that small perturbations in the parameter induce changes in model-observation error metrics which can be resolved above the nondeterministic effect. In practice therefore, the non-deterministic component places a limit on the optimal parameter resolution which can be achieved by model calibration. We find that this limit depends on both the choice of error metric used to compare the model with observations, and the value of the model eddy viscosity parameter. The most robust error metric with respect to the non-deterministic behaviour is the mean kinetic power density over the ADCP deployment period. For a model eddy viscosity $\nu=1 \mathrm{~m}^{2} \mathrm{~s}^{-1}$, the threshold resolvable Manning coefficient is $\mathcal{O}\left(10^{-3}\right) \mathrm{s} \mathrm{m}^{-1 / 3}$, whereas for $\nu=40 \mathrm{~m}^{2} \mathrm{~s}^{-1}$, the threshold decreases to $\mathcal{O}\left(10^{-7}\right) \mathrm{s} \mathrm{m}^{-1 / 3}$. However, the mean power density itself is relatively insensitive to $\nu$. A pragmatic approach to model calibration is therefore to employ high viscosity while calibrating with respect to the friction coefficient. Furthermore, through the use of a variety of meshes within this study, we have found that the optimal parameters do not depend strongly on the mesh resolution, and therefore that this calibration at high viscosity can be performed at coarse resolution, thus saving computational cost. Subsequent mesh refinement and reduction in viscosity can then be used to improve model performance, and attain good agreement with observations via other metrics, such as the flow variability.

This study has used observation data from a single ADCP deployed in the Inner Sound of the Pentland Firth, and considered only spatially uniform model parameters. In future work, we will utilise data from a variety of sources for the purpose of calibrating the model with respect to a spatially varying bottom friction coefficient.

\section{Acknowledgements}

The authors would like to thank SIMEC Atlantis Energy for sharing bathymetry and ADCP data. We acknowledge funding from EPSRC under grants EP/R029423/1 and EP/R511547/1, and additionally acknowledge the Research Computing Service at Imperial College London for HPC resources and support. 


\section{References}

ABPmer. Quantification of Exploitable Tidal Energy Resources in UK Waters, 2007. Technical Report No. 1349, ABP Marine Environmental Research, commissioned by npower Juice.

T. A. A. Adcock, S. Draper, G. T. Houlsby, A. G. L. Borthwick, and S. Serhadlığlu. The available power from tidal stream turbines in the Pentland Firth. Proceedings of the Royal Society A: Mathematical, Physical and Engineering Sciences, 469(2157):20130072, 2013.

T. A. A. Adcock, S. Draper, and T. Nishino. Tidal power generation-a review of hydrodynamic modelling. Proceedings of the Institution of Mechanical Engineers, Part A: Journal of Power and Energy, 229(7):755-771, 2015.

A. Avdis, A. S. Candy, J. Hill, S. C. Kramer, and M. D. Piggott. Efficient unstructured mesh generation for marine renewable energy applications. Renewable Energy, 116:842-856, 2018.

S. Baston, R. E. Harris, D. K. Woolf, R. A. Hiley, and J. C. Side. Sensitivity analysis of the turbulence closure models in the assessment of tidal energy resource in orkney. In 10th European Wave and Tidal Energy conference, Aalborg, Denmark, 2013.

H. Chen, A. Cao, J. Zhang, C. Miao, and X. Lv. Estimation of spatially varying open boundary conditions for a numerical internal tidal model with adjoint method. Mathematics and Computers in Simulation, 97:14-38, mar 2014. ISSN 0378-4754. doi: 10.1016/J.MATCOM.2013.08.005.

Crown Estate. Pentland Firth and Orkney Waters Enabling Actions Report, 2012.

Crown Estate. Pentland Firth and Orkney waters strategic area review project, 2013.

P. F. Cummins and L.-Y. Oey. Simulation of barotropic and baroclinic tides off northern british columbia. Journal of Physical Oceanography, 27(5):762-781, 1997.

Digimap. Marine Themes Digital Elevation Model 6 Arc Second [ASC geospatial data], Scale 1:250000, Tiles: 2062000000, 2060010060, 2060010040, 2060010020, 2060000000, 2058010080, 2058010060, 2058010040, 2058010020, 2058000000, 2056010080, 2056010060, 2056010040, 2056010020, 2056000020, 2056000000, Updated: 25 October 2013, OceanWise, Using: EDINA Marine Digimap Service, https://digimap.edina.ac.uk, Downloaded: 2020-05-29, 2013.

S. Draper, T. A. A. Adcock, A. G. L. Borthwick, and G. T. Houlsby. Estimate of the tidal stream power resource of the Pentland Firth. Renewable Energy, 63:650-657, 2014.

M. C. Easton, D. K. Woolf, and P. A. Bowyer. The dynamics of an energetic tidal channel, the Pentland Firth, Scotland. Continental Shelf Research, 48:50-60, 2012.

G. D. Egbert and S. Y. Erofeeva. Efficient inverse modeling of barotropic ocean tides. Journal of Atmospheric and Oceanic Technology, 19(2):183-204, 2002. doi: 10.1175/1520-0426(2002)019〈0183:EIMOBO $\rangle 2.0 . C O ; 2$.

EMODnet Bathymetry Consortium. EMODnet Digital Bathymetry (DTM 2018). 2018. https://www.emodnetbathymetry.eu/. 
C. Geuzaine and J. F. Remacle. Gmsh: A 3-D finite element mesh generator with built-in pre- and post-processing facilities. International Journal for Numerical Methods in Engineering, 79(11):1309-1331, 2009. doi: 10.1002/nme. 2579 .

P. A. Gillibrand, R. A. Walters, and J. McIlvenny. Numerical simulations of the effects of a tidal turbine array on near-bed velocity and local bed shear stress. Energies, 9(10):852, 2016.

L. Goddijn-Murphy, D. K. Woolf, and M. C. Easton. Current patterns in the Inner Sound (Pentland Firth) from underway ADCP data. Journal of Atmospheric and Oceanic Technology, 30(1):96-111, 2013.

A. W. Heemink, E. E. A. Mouthaan, M. R. T. Roest, E. A. H. Vollebregt, K. B. Robaczewska, and M. Verlaan. Inverse 3D shallow water flow modelling of the continental shelf. Continental Shelf Research, 22(3):465-484, feb 2002. ISSN 0278-4343. doi: 10.1016/S0278-4343(01)00071-1.

N. Huybrechts, H. Smaoui, S. Orseau, P. Tassi, and F. Klein. Automatic Calibration of Bed Friction Coefficients to Reduce the Influence of Seasonal Variation: Case of the Gironde Estuary. Journal of Waterway, Port, Coastal, and Ocean Engineering, 147(3):05021004, 2021.

O. P. Jones, O. S. Petersen, and H. Kofoed-Hansen. Modelling of complex coastal environments: Some considerations for best practise. Coastal Engineering, 54(10):717-733, 2007.

T. Kärnä, B. De Brye, O. Gourgue, J. Lambrechts, R. Comblen, V. Legat, and E. Deleersnijder. A fully implicit wetting-drying method for DG-FEM shallow water models, with an application to the Scheldt Estuary. Computer Methods in Applied Mechanics and Engineering, 200(5-8):509-524, 2011.

T. Kärnä, S. C. Kramer, L. Mitchell, D. A. Ham, M. D. Piggott, and A. M. Baptista. Thetis coastal ocean model: discontinuous Galerkin discretization for the three-dimensional hydrostatic equations. Geoscientific Model Development, 11(11):4359-4382, 2018.

M. Kreitmair, T. Adcock, A. Borthwick, S. Draper, and T. van den Bremer. The effect of bed roughness uncertainty on tidal stream power estimates for the Pentland Firth. Royal Society Open Science, 7(1):191127, 2020.

M. J. Kreitmair, S. Draper, A. G. L. Borthwick, and T. S. Van Den Bremer. The effect of uncertain bottom friction on estimates of tidal current power. Royal Society open science, 6(1):180941, 2019.

$\mathrm{X}$. Lu and J. Zhang. Numerical study on spatially varying bottom friction coefficient of a 2D tidal model with adjoint method. Continental Shelf Research, 26(16):1905-1923, oct 2006. doi: 10.1016/J.CSR.2006.06.007.

D. MacKay. Sustainable Energy - without the hot air. UIT cambridge, 2008.

Marine Scotland. Pentland firth bathymetric package, 2013.

Marine Scotland. Pilot Pentland Firth and Orkney Waters Marine Spatial Plan, Socio-Economic Baseline Review, 2015.

S. Maßmann. Sensitivities of an adjoint, unstructured mesh, tidal model on the European Continental Shelf. Ocean Dynamics, 60(6):1463-1477, dec 2010. ISSN 16167341. doi: 10.1007/s10236-010-0347-6. 
S. P. Neill, A. Vögler, A. J. Goward-Brown, S. Baston, M. J. Lewis, P. A. Gillibrand, S. Waldman, and D. K. Woolf. The wave and tidal resource of Scotland. Renewable energy, 114:3-17, 2017.

R. B. O'Hara Murray and A. Gallego. Data review and the development of realistic tidal and wave energy scenarios for numerical modelling of Orkney Islands waters, Scotland. Ocean \& Coastal Management, 147:6-20, 2017.

R. B. O'Hara Murray and A. Gallego. A modelling study of the tidal stream resource of the Pentland Firth, Scotland. Renewable energy, 102:326-340, 2017.

W. Pan, S. C. Kramer, T. Kärnä, and M. D. Piggott. Comparing non-hydrostatic extensions to a discontinuous finite element coastal ocean model. Ocean Modelling, 151:101634, 2020.

B. L. Polagye, J. Epler, and J. Thomson. Limits to the predictability of tidal current energy. In $O C E A N S$ 2010 MTS/IEEE SEATTLE, pages 1-9. IEEE, 2010.

A. Rahman and V. Venugopal. Inter-comparison of 3d tidal flow models applied to orkney islands and pentland firth. In Proceedings of the 11th European Wave and Tidal Energy Conference (EWTEC 2015), Nantes, France, pages $6-11,2015$.

A. Rahman and V. Venugopal. Parametric analysis of three dimensional flow models applied to tidal energy sites in Scotland. Estuarine, Coastal and Shelf Science, 189:17-32, 2017.

F. Rathgeber, D. A. Ham, L. Mitchell, M. Lange, F. Luporini, A. T. T. McRae, G.-T. Bercea, G. R. Markall, and P. H. J. Kelly. Firedrake: automating the finite element method by composing abstractions. ACM Transactions on Mathematical Software (TOMS), 43(3):1-27, 2016.

M. A. Shields, L. J. Dillon, D. K. Woolf, and A. T. Ford. Strategic priorities for assessing ecological impacts of marine renewable energy devices in the Pentland Firth (Scotland, UK). Marine Policy, 33(4):635-642, 2009.

J. Side, A. Gallego, M. A. James, I. Davies, M. Heath, H. Karunarathna, V. Venugopal, A. Vögler, and M. Burrows. Developing methodologies for large scale wave and tidal stream marine renewable energy extraction and its environmental impact: An overview of the TeraWatt project. Ocean and Coastal Management, 2017.

I. Sraj, M. Iskandarani, A. Srinivasan, W. C. Thacker, J. Winokur, A. Alexanderian, C.-Y. Lee, S. S. Chen, and O. M. Knio. Bayesian inference of drag parameters using AXBT data from Typhoon Fanapi. Monthly Weather Review, 141(7):2347-2367, 2013.

I. Sraj, M. Iskandarani, W. C. Thacker, A. Srinivasan, and O. M. Knio. Drag parameter estimation using gradients and hessian from a polynomial chaos model surrogate. Monthly Weather Review, 142(2):933-941, 2014a.

I. Sraj, K. T. Mandli, O. M. Knio, C. N. Dawson, and I. Hoteit. Uncertainty quantification and inference of Manning's friction coefficients using DART buoy data during the Tōhoku tsunami. Ocean Modelling, 83:82-97, 2014b.

C. V. M. Vouriot, A. Angeloudis, S. C. Kramer, and M. D. Piggott. Fate of large-scale vortices in idealized tidal lagoons. Environmental Fluid Mechanics, 19(2):329-348, 2019. 
S. Waldman, S. Baston, R. Nemalidinne, A. Chatzirodou, V. Venugopal, and J. Side. Implementation of tidal turbines in MIKE 3 and Delft3D models of Pentland Firth \& Orkney Waters. Ocean 83 Coastal Management, 147:21-36, 2017.

S. C. Warder and M. D. Piggott. Optimal experiment design for bottom friction parameter estimation. Submitted to Ocean Modelling, 2021. doi: https://doi.org/10.31223/X5CG79.

P. Wessel and W. H. F. Smith. A global, self-consistent, hierarchical, high-resolution shoreline database. Journal of Geophysical Research: Solid Earth, 101(B4):8741-8743, 1996. doi: 10.1029/96JB00104.

J. Wolf, , C. Stuiver, D. Price, H. Johnson, A. Gallego, and R. B. O'Hara Murray. The Scottish shelf model. Part 2: Pentland Firth and Orkney waters sub-domain. Scottish Mar. Freshw. Sci, 7(4):248, 2016.

J. Zhang, X. Lu, P. Wang, and Y. P. Wang. Study on linear and nonlinear bottom friction parameterizations for regional tidal models using data assimilation. Continental Shelf Research, 31(6):555-573, apr 2011. doi: 10.1016/J. CSR.2010.12.011. 